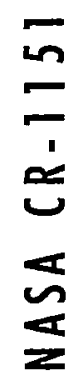

NASA CONTRACTOR REPOR T

LOAN COPY: RETURN TO

AFWL (WLIL-2)

WIRTLAND AFB, N IMEX

\section{THE HUMAN AS AN OPTIMAL CONTROLLER AND INFORMATION PROCESSOR}

by Sheldon Baron and David L. Kleinman

Prepared by

BOLT BERANEK AND NEWMAN, INC.

Cambridge, Mass.

for Electronics Research Center

NATIONAL AERONAUTICS AND SPACE ADMINISTRATION - WASHINGTON, D. C. - SEPTEMBER 1968 
THE HUMAN AS AN OPTIMAL CONTROLLER AND INFORMATION PROCESSOR

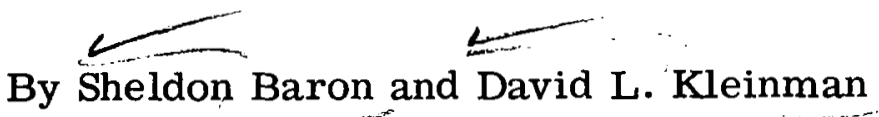

Distribution of this report is provided in the interest of information exchange. Responsibility for the contents resides in the author or organization that prepared it.
$3 B$ -
- Sisued by Originator as Report-Ne: 1571

Prepared under Contract No. NAS 12-104 by

BOLT BERANEK AND NEWMAN, INC.

Cambridge, Mass.

for Electronics Research Center

NATIONAL AERONAUTICS AND SPACE ADMINISTRATION

For sale by the Clearinghouse for Federal Scientific and Technical Information Springfield, Virginia 22151 - CFSTI price $\$ 3.00$ 
TABLE OF CONTENTS

Page

SUMMARY

1

INTRODUCTION

3

SYMBOLS

VISUAL SAMPLING MODELS: A BRIEF REVIEW

$\begin{array}{ll}\text { HUMAN OPERATOR MODEL } & 17\end{array}$

$\begin{array}{ll}\text { Vehicle Dynamics and Display } & 18\end{array}$

Perceptual Processor 19

Controller and Data Reconstructor 22

Sampler 24

Summary 25

$\begin{array}{ll}\text { OPTIMAL SAMPLING PROBLEM } & 27\end{array}$

Mathematical Formulation $\quad 28$

Solving the Optimal Sampling Problem 36

$\begin{array}{ll}\text { Summary and Comments } & 38\end{array}$

A SIMPLE EXAMPLE 43

A Two-Axis Tracking Problem 43

Choice of Sampling Parameters 44

Optimal Sampling Problem for Two-Axis Tracking 46

$\begin{array}{ll}\text { Results and Discussion } & 48\end{array}$

CONCLUSION 65

$\begin{array}{ll}\text { Some Topics for Further Investigation } & 67\end{array}$

$\begin{array}{ll}\text { REFERENCES } & 69\end{array}$ 
LIST OF ILLUSTRATIONS

Figure

Page

1

Smallwood's Block Diagram of the Human Operator

10

2

Model Structure

21

3 Data Reconstructor

21

4 Effect of Controlled Element Dynamics on

Fractional Time Spent on Instrument 1

4b Effect of Controlled Fiement Dynamics on Fractional Time Spent on Instrument 2

5 Effect of Controlled Element Dynamics on Optimal Sampling Period

6 Effect of Controlled Element Dynamics on Minimum "Sampling Cost"

7 Effect of Observation Noise on Optimal Sampling, Period

Effect of Observation Nolse on "Sampling Costs"

Effect of Input Noise Covariance on Sampling Period 61

10

Effect of Input Noise Covariance on Fractional

Allocation of Attention

11 Effect of Control Requirements on Fractional Time Spent on Instrument 1 


\section{THE HUMAN AS AN OPTIMAL \\ CONTROLLER AND INFORMATION PROCESSOR \\ by \\ Sheldon Baron and David L. Kleinman}

\section{SUMMARY}

A mathematical model of the human operator in multivariable control tasks is developed by considering the human as a control and information-processing system. The model contains elements for describing the operator's inherent physiological limitations as well as his instrument-monitoring, data-reconstruction and control behavior. Special emphasis is placed on the instrument-monitoring aspects of the model.

The human's limitations are modelled by combining them into an equivalent perceptual time delay and an equivalent observation nolse. The main assumption underlying the subsequent theoretical investigations is that the well-trained, well-motivated operator behaves in a near optimal manner, subject to the constraints imposed by the above limitations. Thus, the operator's control behavior is assumed to be that of an ideal feedback controller. The human's data-reconstruction process is chosen so as to obtain a "best" estimate of the state of the controlled element based on information obtained from "sampling" the various instruments. The data reconstructor consists of a Kalman estimator and a predictor in tandem, its structure is fixed but it depends, parametrically, on the sampling behavior.

Instrument-monitoring behavior depends explicitly on the control task and on the control actions. Provision is made for the ability to obtain information from the peripheral visual field and 
there are no restrictions on signal coupling. The visual sampling model also includes means for constrainine instrument scanning rates. The specific characteristics of the operator's visual sampling behavior are predicted by solving a nonlinear optimization problem. This problem is precisely formulated and methods for its solution are discussed. By changing the variances of the observational noises it is possible to predict the effects that changes in the visual display panel will have upon the human's sampling, behavior. Finally, instrument sampling, characteristics for a simple two-axis compensatory tracking task are obtained. The results exhibit the general characteristics one would expect from a human operator performing a similar task. 


\section{INTRODUCTION}

The basic objective of research to be performed under Contract No. NAS-12-104 is the investigation of representations of the human operator with a view towards their relation to display system evaluation. Our previous efforts under this contract were primarily concerned with developing new methods for identifying the characteristics of human pilot control behavior. In that work we examined linear and nonlinear representations of the pllot in a single-axis compensatory tracking task (Ref. 1). Such models are useful for obtaining a better understanding of pilot control behavior and, hence, for synthesizing manual control systems. However, since the models were developed for single-axis tracking and required no visual sampling, they represented only a first step in the development of analytic display evaluation techniques.

We are now investigating the problem of manual control in more complex, multivariable situations. Our goal in this phase of the research is to obtain a more complete mathematical description of the human operator's information-processing and control behavior. (It should be understood that our aim is not necessarily to describe the conscious thought processes or physical structure of the operator; rather, we hope to model his overt behavior. Thus the models will be valid to the extent that their input-output behavior is analogous to that of the human operator.)

In this report we shall describe our model of the human operator as an information-processor and controller. We emphasize those aspects of the model concerning visual sampling of multiinstrument display panels as this represents the major thrust of our recent and current research under this contract. We begin with a brief review of past research in human instrument sampling. 
Then we describe the structure of our model of human operator behavior in a multivariable control task and show how the sampling model interfaces with other elements of the overall human operator model. The model we have postulated is rooted in modern control theory and, as we shall see, to obtain the model parameters (e.g., control gains, sampling strategies, etc.), we must solve a complex optimization problem. The theoretical background for this problem is discussed and we then give a precise mathematical formulation of the optimization problem. Numerical results for a simple, two axis problem are obtained and these results serve to 1llustrate various aspects of the problem. Finally, we discuss implications of our research efforts and the directions for further work. 


\section{SYMBOLS}

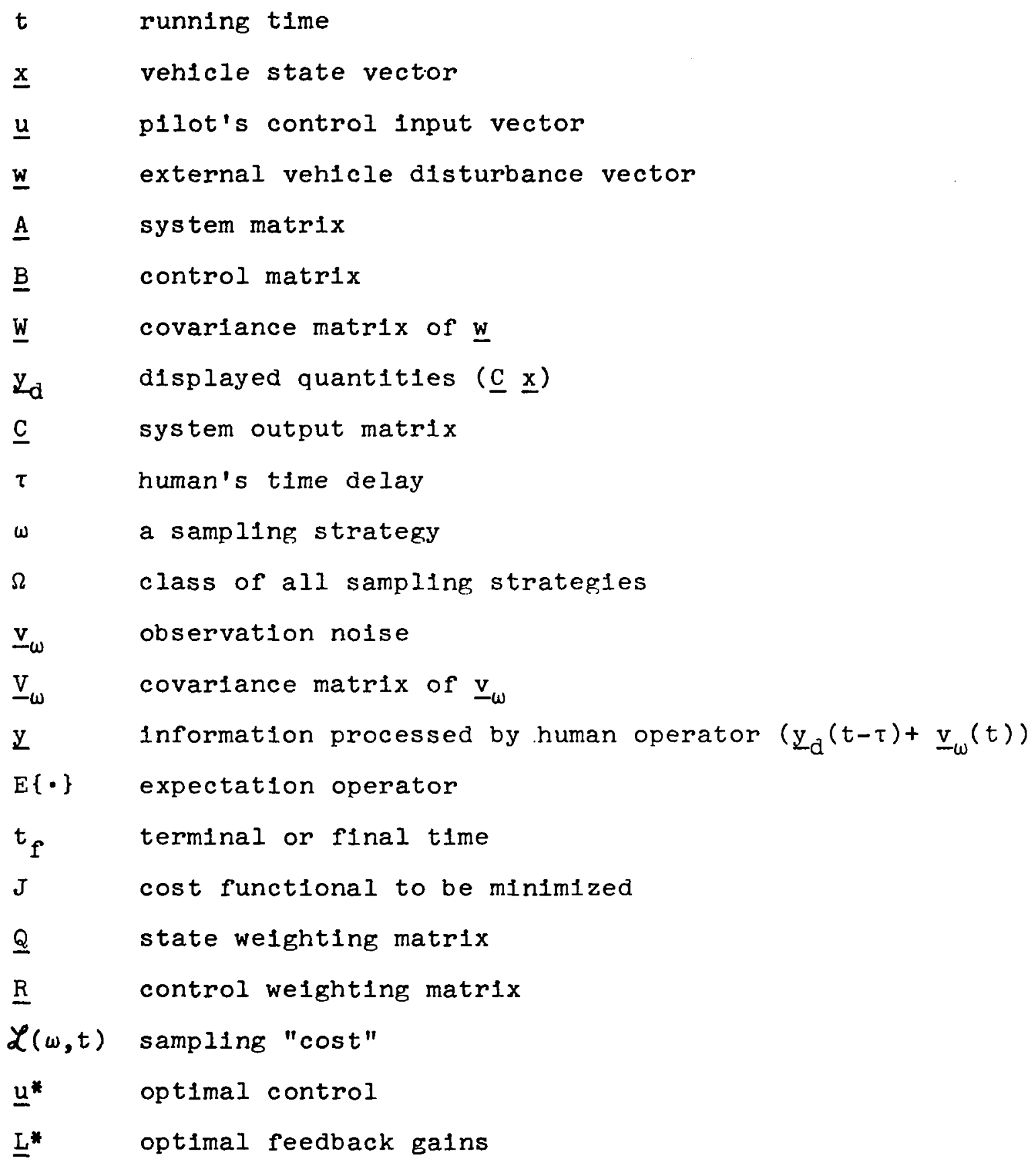




\section{SYMBOLS (Continued)}

$\begin{array}{ll}\hat{\underline{x}}_{\omega} & \text { least mean squared estimate of } \underline{x} \\ J^{*} & \text { minimum cost } \\ \omega^{*} & \text { optimaI sampling strategy } \\ \hat{\Omega} & \text { range space for function } \omega(\cdot) \varepsilon \Omega \\ \underline{K} & \text { time varying solution of matrix Riccati equation } \\ \bar{K} & \text { asymptotic Riccati Equation } \\ \underline{\Sigma} & \text { "error" covariance matrix } \\ I(\omega) & \text { part of } J^{*} \text { which depends explicitly on } \omega \\ T & \text { sampling period } \\ \underline{\Sigma} & \text { asymptotic variance equation } \\ T^{*} & \text { optimal sampling period }\end{array}$




\section{VISUAL SAMPLING MODELS: A BRIEF REVIEW}

Although a detalled understanding of human visual sampling processes in a complex control or monitoring task is essential to the development of rational display design procedures, there has been surprisingly little research in this area of human engineering. The first significant attempt to understand how pilots use their eyes in order to obtain information from many separate instruments was made at Wright-Patterson Air Force Base in the period 1949-1954 (Refs. 2-10). The WPAFB studies were aimed at determinIng patterns of pilot eye movements in actual control situations. Eye fixations of pilots were recorded in a series of flight tests and the frequency and duration of fixations on various instruments, as well as transitions between palrs of instruments, were determined for a number of flight conditions. However, no attempt was made in these studies to model the human visual sampling process. Indeed, the inputs to the instruments were not recorded and, consequently, the data is of limited utility with respect to verifying any predictive models.

The first quantitative model for describing pilot sampling behavior was developed by Senders (Ref. 11). This model was based on information-theoretic ideas, particularly Shannon's sampling theorem (Ref. 12). A basic assumption of the model was that the human observer samples the various signals periodically and attempts to reconstruct the time functions presented on each instrument. Moreover, it was assumed that the operator was effectively a single channel device capable of attending to only one signal at a time. With these concepts as a starting point, senders was able to derive expressions for the frequency and duration of samples of an instrument given its input signal characteristics and the required precision of readout. This model predicted quite well the average 
behavior of subjects in an experimental situation. In the experiments the subject's task was to monitor a panel containing four instruments driven by zero-mean gaussian signals of different bandwidths, and to indicate whenever any of the signals exceeded a predetermined threshold. Since the subject's task was quite different than the one assumed in deriving the model, it is somewhat surprising that there was such good agreement between theory and experiment. Senders (Ref. 13) explains this good agreement by noting that the sampling frequencles would be proportional to the signal bandwidths only if the signal power and the magnitudes of significant deviations ${ }^{*}$ were the same for all the signals and that such was the case in his experiments. Thus, the agreement which was obtained was somewhat fortuitous since it depended on a unique experimental condition.

It seemed reasonably clear that the simple periodic sampling model would not adequately predict behavior in more complex situations, especially since observed data gave evidence of aperiodic sampling behavior (Ref. 13). Taking a cue from the fact that pilots often are only concerned with detection of extreme readings rather than with signal reconstruction, Senders proposed a conditional sampling scheme which would result in aperiodic behavior. In this approach, the human monitor is considered as a channel for the transmission of discrete messages and not as a channel for the transmission of a complete time function. In this context it is possible to postulate several, not necessarily mutually exclusive, sampling strategies. Thus, Elkind (Ref. 14) hypothesizes a strategy in which a sample is taken when the probability that the signal exceeds a prescribed limit is greater than some subjective probability threshold, whereas Grignetti (Ref. 14) assumes that a

* Senders defines a significant deviation as an excursion of the signal beyond the predetermined threshold. 
sample is taken when the probability of exceeding the limit is a maximum. Senders (Ref. 14), on the other hand, suggests a sampling strategy based on a "Variable Nyquist Interval".

Unfortunately, none of the above conditional sampling models have been tested agalnst experimental data. Smallwood (Ref. 15), however, has developed a model which has been tested against the data of the simple experiment described above with encouraging results. Although similar in some of the details, Smallwood's model is conceptually quite different from those previously developed. His view of the human operator is portrayed in Figure 1 . The model is based on two underlying assumptions: 1) the human overator bases his state of information about his environment upon an internal model of this environment; the model is formed as a result of past perceptions of his environment; 2) the human operator behaves optimally with respect to his task and his current state of information within his physical limitations. Of course, to apply the model, one must make further assumptions. First, one must postulate a form for the internal model which describes the monitor's conception of the environment he is monitoring. Smallwood's approach to this problem is to assume that the monitor's model of each instrument is a good approximation to the true situation. Thus, in attempting to predict the results of the laboratory experiment described above, the internal model is based upon the assumption that the instrument readings are the result of passing white gaussian nolse through a linear time invariant filter. By allowing higher-order filters, Smallwood is able to account for the perception of signal rate as well as signal amplitude. This was not possible with the previous models. The next step in specifying Smallwood's model is to define what is meant by the statement that the human operator behaves optimally with respect to his task. Smallwood's interpretation is that the human monitor, interested in detecting immediate excursions of the instruments 


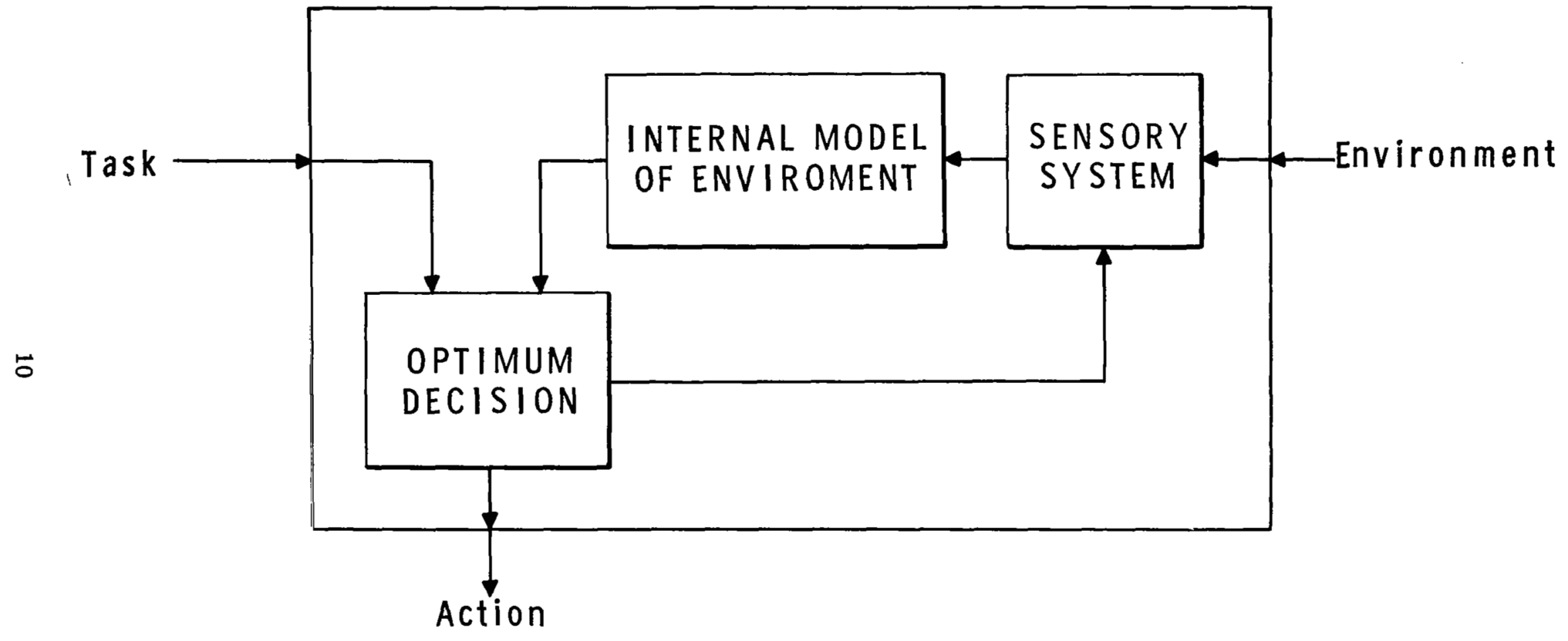

FIG. I SMALLWOOD'S BLOCK DIAGRAM OF THE HUMAN OPERATOR 
beyond the threshold, switches his attention to that instrument for which the probability of exceeding the threshold is a maximum. Note that this decision criterion is quite similar to those of Elkind and Grignetti.

The last aspect of Smallwood's model which requires specification is the operator's sensory system. The following assumptions. are made by Smallwood in arriving at a model for the human visual perception process.

(1) A "dead time" of 0.1 seconds is required to shift attention between two different instruments.

(2) Upon fixating on an instrument a record of the instrument reading is stored instantaneously within the short-term memory.

(3) The readout time is dependent upon the precision of the required reading, and this precision is dependent in turn upon the proximity of the instrument reading to one of the thresholds.

As we have noted previously, there is very little experimental data with which to compare the predictions of the above models. Even in the cases where such comparisons can be made, 1.e., Senders and Smallwood's predictions for the sampling behavior in the laboratory experiments, the experimental situation was such that it is virtually impossible to make inferences concerning the general "operational" validity of these models. However, it is possible to point out some inherent limitations in these models. The first and perhaps most significant limitation is that none of the models have been used to take into account, in any meaningful way, correlation between various instruments. Senders (Ref. 14) made a preliminary attempt to include correlation between instruments and went so far as to run laboratory experiments with correlated signals. However, the results from the experiment were far from encouraging. Smallwood's model does not appear 
to have any fundamental limitations with regard to signal correlation. In fact, with the appropriate choice for an internal model, it seems fairly clear that a method for dealing with coupling is possible within the framework of his model, but he has, thus far, eschewed any attempt to include such effects. A second significant Iimitation of these models is that none of them take into account, explicitly, the interaction between sampling and control behavior. In all cases the human operator is considered as a monitor only; the control requirements play little if any role in the selection of a sampling strategy. It is true that the a posteriori signal analysis which is necessary to obtain the parameters of the conditional sampling models will include the effects of control. However, this is quite different from using the control task in an attempt to predict sampling behavior. Again, Smallwood's model seems least limited with regard to including such possibilities; but, as in the case of signal coupling, Smallwood has not made any attempt to do so.

All of the models consider the human operator as a singlechannel device capable of processing information from only one instrument at a time. This approach is at variance with the recent experimental data, obtained by Levison and Elkind (Ref. 16), concerning peripheral tracking. Their experiments showed that under certain conditions pilots could perform two-axis compensatory tracking even though the information concerning one of the axes was always in the peripheral visual field. While there might be some argument as to whether the pilot is actually processing both signals simultaneously, it seems fairly clear that the ability to perform peripheral tracking will in fact affect the pilot's sampling behavior and should therefore be accounted for in a sampling model. Still another limitation of the models is that no risk or cost structure has been incorporated. Equal costs are assigned to all instruments. Moreover, there is no cost assigned 
to taking a sample or to switching attention. It is possible within the context of the Senders-type models to implicitly allow different costs by assigning different allowable errors in readout. However, such an approach is qualitative at best. Smallwood assumes a dead time for shifting of attention between two different instruments in his model; this dead time may be interpreted as a cost for switching attention. Finally, we note that the Senders, Elkind and Grignetti models all assume that the instruments are fed with zero-mean gaussian signals.

Carbonell (Ref. 17) has attempted to overcome some of the above limitations by developing a model of visual sampling which has its roots in queuing theory. If the human is assumed to be a single-channel processor, then one is led to the notion that the various information sources, $1 . e$. , instruments, queue-up and wait their turn to be processed. The analysis of sampling can then be approached as a problem in queuing theory and one can arrive at estimates of the probability distribution of simultaneous demands, the probability distribution of waiting times and estimates of the probability that events of interest will be missed. To be more explicit, Carbonell assumes the following:

(1) Each time the observer looks at one instrument, he is postponing the observation of others.

(2) The observer makes an intelligent decision before looking at an instrument each time, 1.e., he tries to minimize the risk involved in not observing the other instruments.

(3) This risk is represented by the probability that the readings may, while not being observed, exceed a certain threshold leading to some catastroph1c result (such as negative altitude or lack of fuel). 
(4) The time involved in reading each instrument will be assumed constant (of the order of $1 / 3$ of a second) for all instruments. If the observer looks at one instrument for a longer time, it is considered as a second (third, etc.) consecutive reading of the same instrument. In other words, the observer chooses this instrument again to minimize his total cost.

(5) The observer's task in visually sampling his instruments is part of a feedback loop closed through his control actions.

As can be seen from his assumptions, Carbonell has Indeed addressed himself to some of the limitations of the previous models. In particular he includes a cost structure and the possibility of control actions; in regard to the latter, it is only fair to point out that he only accounts for control in the most rudimentary way. * Although not apparent from the assumptions, Carbonell also removes the restriction of zero-mean gaussian signals. Instead, he assumes that, in the case of no control, the signals are generated by a sort of random walk process with equal probability of being above or below the value last read by increments that are gaussianly distributed. He assumes further that it is only through control action that the process can be brought to equilibrium, that is, that the long-term signal will be zero mean. However, even in such a case, the overall result is not a zero mean gaussian signal. Having made these assumptions, it still remains necessary to specify a queue discipline in order to apply Carbonell's model. This is an extremely difficult task in view of the highly nonlinear decision process involved. In order to overcome this difficulty, Carbonell decided to simulate the problem on a digital computer and he has obtained some interesting

\footnotetext{
* He assumes that if control is effected that it is of the form $\exp (-k t)$.
} 
results. In particular, the simulation revealed that his model resulted in periodic sampling only when there was a set of low varlance instruments and a relaxed task. Larger variances and, hence, harder tasks resulted in progressively more aperiodic sampling. This result agrees well with the observed sampling behavior of human pilots in corresponding circumstances.

Carbonell's model is clearly more general and more flexible than any of the other models previously developed. Nevertheless it has not removed all the previously cited limitations. Peripheral processing is not accounted for; indeed, the concept of the human operator being a single-channel processor is central to the idea of the instrument queue. Also, coupling among instruments is not presently included in his model, although he claims that such coupling could be incorporated. Finally, it should be pointed out that Carbonell pays a heavy price in analytic complexity for the flexibility that he has obtained. It appears that only through extensive simulation can one obtain the pertinent model parameters and predict human sampling behavior.

We close our review of visual sampling models by noting that the models of Senders (perlodic sampler), Smallwood and Carbonell are currently being tested for their adequacy to predict behavior in a more complex experimental situation (Ref. 18). Pilot eyemovements were recorded during maneuvers "flown" on a Link CllB Trainer. Signals driving the instruments were also recorded so that they could be analyzed in conjunction with the eye-movement data. Unfortunately, the results of the analysis are not yet complete and, hence, no conclusions can be drawn at the present time. 
$\mid$ 
HUMAN OPERATOR MODEL

In this chapter we discuss the structural form of our model of the human operator as a control and information-processing system. Since the problems of interest are generally multivariable, state-space techniques and modern control theory are most suited to their study and, hence, will play a central role in the development. The basic assumption underlying our approach is that the well-motivated, well-tralned human operator behaves in a nearoptimal manner, subject to his inherent limitations and constraints. This is not a new idea. Several researchers have attempted to apply optimization theory to the development of models of human control behavior. The most recent attempt was made by Elkind, et al ${ }^{*}$ (Ref. 19). They used modern optimization theory to predict human behavior in a multivariable tracking task, corresponding to a $\mathrm{V} / \mathrm{STOL}$ vehicle in hover. The results of that study were quite promising and our model of controller behavior represents a further development of their approach. As our discussion of the previous chapter shows, various optimization techniques have also been applied to the problem of visual sampling. Indeed, the models of Senders, Smallwood and Carbonell are all based on some optimization concept. However, as we shall see, our approach to the optimization of visual sampling shall be quite different from the ones taken by those investigators. Moreover, we shall be concerned with the combined control and information processing problem.

The human operator model which was adopted in this study is shown in Figure 2 ( $p, 21)$. The model includes some of the human's

\footnotetext{
* A brief summary of other attempts to predict human controller characteristics via optimization techniques is given in Ref. 19.
} 
physical limitations as well as representations for his control and information processing behavior. Its basic structure has much in common with Smallwood's model of Figure 1. The displayed state $y_{d}$ is essentially a representation of the human operator's environmental input. The block labeled perceptual processor is related to Smallwood's sensory system and, as we shall see, the data reconstructor is comparable to Smallwood's internal model.

We now clarify some of these ldeas by briefly describing each element in the pilot-vehicle display model of Figure 2.

Vehicle Dynamics and Display--The human operator's basic task is to control, in some prescribed way, a dynamic vehicle. Several vehicle outputs may be of concern and the operator has several inputs through which he can exert control on the vehicle. It is assumed that the only means avallable for monitoring the vehicle's performance is a display panel consisting of several instruments. No external visual or kinesthetic cues are avallable.

We shall assume that the vehicle dynamics are adequately represented by the linear equations of motion

$$
\underline{\dot{x}}(t)=\underline{A} \underline{x}(t)+\underline{B} \underline{u}(t)+\underline{w}(t)
$$

where $\underline{x}$ is the vehicle state, $\underline{u}$ the pllot's control input, and where $w$ represents the external disturbances (e.g., wind gusts). For analytic tractability, $\underline{w}(t)$ will be considered a zero-mean, gaussian white noise with covariance $\underline{W}$.

We further assume that displayed variables are linear combinations of the vehicle states. Thus, we account for the fact that all of the vehicle states may not be explicitly displayed. As an interesting sidelight, we note that such a representation may allow us to investigate such things as integrated displays or the presentation of quickened information. 
Perceptual Processor--The human operator has certain physical limitations which prevent him from making a perfect reading, except in the simplest of cases, of the value presented on an instrument. In the case of many instruments, it is clear that he can not make simultaneous, perfect observations. Moreover, even if he were able to make such observations, his limitations prevent him from taking instantaneous and precise action. These limitations must be accounted for in any reasonable mathematical model of the human operator. We now briefly discuss these limitations and our method for including them in the model.

The inherent limitations of the human operator are manifold and complex. However, as a first approximation, the number of limitations to be considered can be reduced by combining phenomena which have similar effects. For example, the time delays associated with visual, central processing and neuro-motor pathways can be combined and represented by an equivalent time-delay which is associated with perception. In addition, neuro-muscular dynamics can be approximated by a delay (Ref. 20) which can also be combined with the neuro-delays and treated as an equivalent perceptual delay. Similarly, since preliminary analysis has shown that It will be very difficult to distinguish experimentally between observation noise, motor noise, and certain types of time variations in control strategy, we can represent all three factors as a single equivalent "observational noise" process. * The human operator's ability to make precise measurements is, however, a function of many factors. The visual environment (ambient light levels, glare, etc.) affects his ability to measure variables.

For example, instruments of an on-off nature.

Indeed, we can include "measurement noise" associated with the vehicle's sensors and with the displays themselves in the "observation nolse." 
of more importance to this study, the ability to measure precisely a quantity would depend on the type and form of the display and on the location of the display in the visual field, that is, on whether the display is being viewed foveally or peripherally. With the above comments in mind, we assume that the perceptual process can be modelled by an equation of the form

$$
\underline{y}(t)=\underline{y}_{d}(t-\tau)+\underline{v}_{\omega}(t)=\underline{C} \underline{x}(t-\tau)+\underline{v}_{\omega}(t)
$$

where $\underline{y}(t)$ is the "observation" processed by the human operator, $\underline{y}_{d}(s)$ is the displayed state, $\tau$ is a delay and $\underline{v}_{\omega}(t)$ is "observational noise". (Note that $\underline{y}_{d}(s)=\underline{c} \underline{x}(s)$, as described previously.)

We shall assume that the observational noise $\underline{v}_{\omega}$ is a gaussian white noise process with a covariance $\underline{V}_{\omega}$ that depends on the sampling strategy $\omega$. The white noise assumption is, to an extent, physically unreasonable but it is a good approximation to an observational noise process that is wideband with respect to the system bandwidth. Numerical values for the covariance will depend on where the pilot is looking (i.e., whether he is viewing an instrument peripherally or foveally) and on relevant features of the display panel. Precise quantitative determination of these values is, of course, an extremely difficult task and it is not presently clear exactly how they will be measured. However, as we shall see, in some instances a coarse approximation to the values of $\underline{v}_{\omega}$ may be sufficient. The method we have used for representing the perceptual process makes the choice of a sultably descriptive class of allowable sampling strategies an important aspect of the problem. However, before turning our attention to the determination of sampling behavior, let us examine the control and data reconstruction portions of our model. 


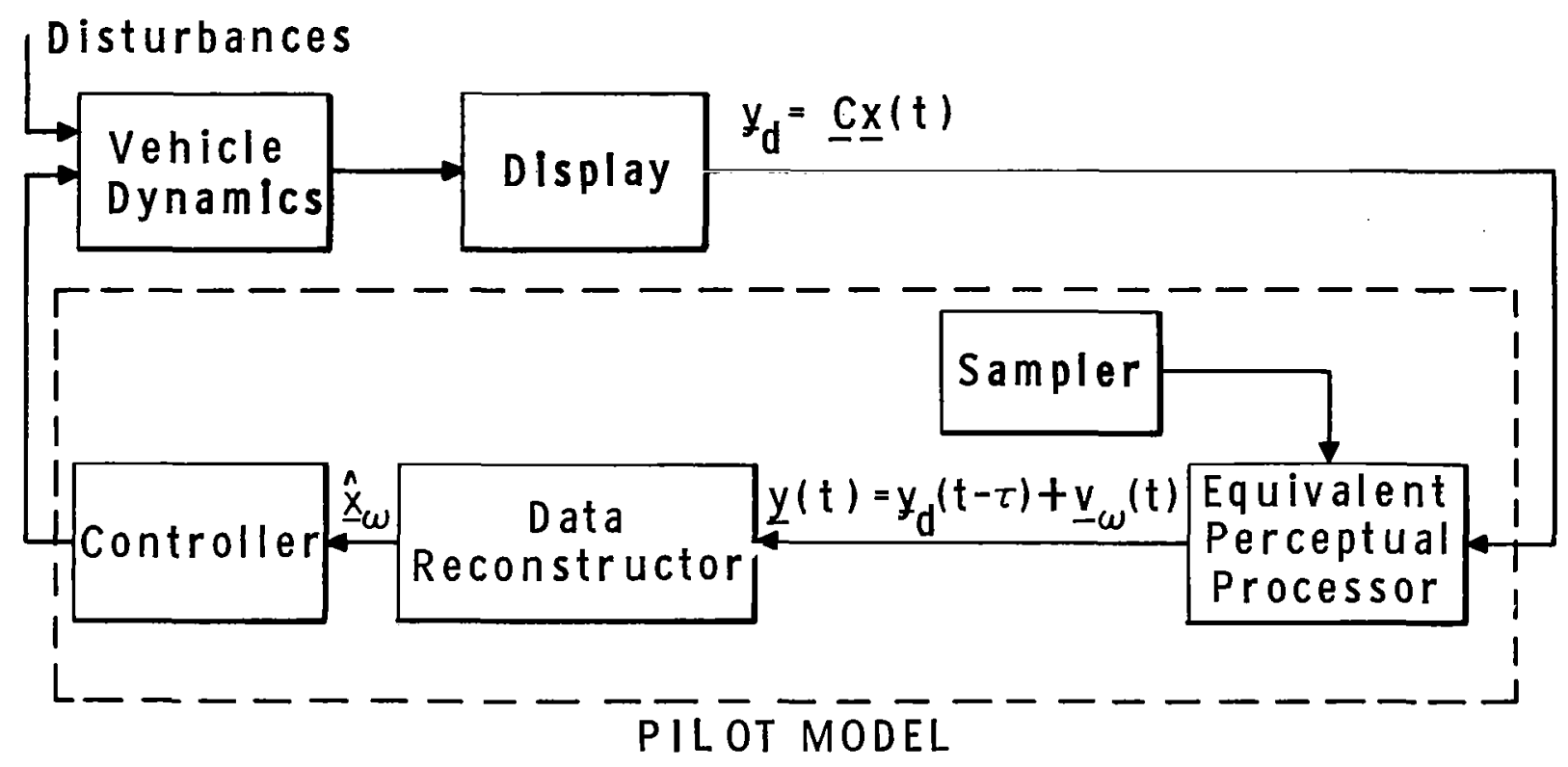

FIG. 2 MODEL STRUCTURE

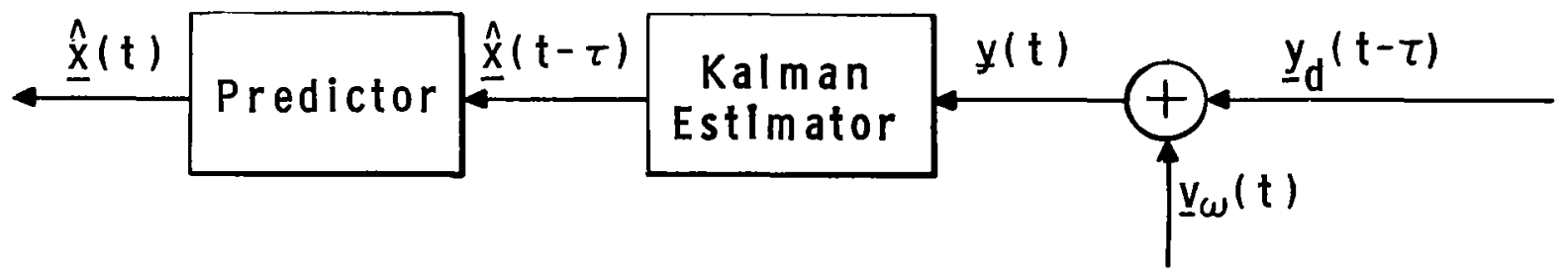

FIG.3 DATA RECONSTRUCTOR 
Controller and Data Reconstructor--In this section we give precise meaning to the human operator's control objectives and develop a model for his resulting control characteristics. We also postulate a model for describing the manner in which the operator reconstructs the data necessary for control from the information obtained by sampling the instruments on the display panel.

We assume that the pllot's control objective is to select an input $\underline{u}(t)$ to the dynamical system (given by Eq. (I)) so as to minimize a cost functional of the form

$$
J(\omega, \underline{u})=E\left\{\lim _{f \rightarrow \infty} \frac{I}{t_{f}} \int_{0}^{t_{f}}\left[\underline{x}^{\prime} \underline{Q} \underline{x}+\underline{u}^{\prime} \underline{R} \underline{u}+\mathcal{L}(\omega, t)\right] d t\right\}(3)
$$

The quantity $\mathscr{L}(\omega, t)$ in the cost functional is included to allow a "direct" cost for sampling. It does not explicitly depend on the control input $\underline{u}$ and thus, assuming a fixed sampling strategy $\omega$, the human controller's characteristics are determined by the solution of an "optimal regulator problem" (Ref. 21). Clearly, not all real control problems can be represented in this way but a great many can be. Moreover, using linear dynamics and specifying a control task of minimizing the cost functional of Eq. (3) represents a natural extension of the classical manual control compensatory tracking experiments. In addition, the resulting analytic simplification is so great as to fustify extensive consideration of problems of this type. The solution of this optimization problem is well known (Ref. 22) and is characterlzed by the linear feedback law

$$
\underline{u}^{*}(\omega)=-\underline{I}^{*} \underline{\hat{x}}_{\omega}(t)
$$


where $\hat{x}_{\omega}(t)$ is the least mean square estimate of the state $x(t)$, given the observed data $\underline{y}(\tau), \tau \leq t$. The feedback gains $\underline{L}^{*}$ are independent of $\omega$ and depend on the weighting matrices $\underline{Q}$ and $\underline{R}$. The minimum cost is denoted by

$$
J^{*}(\omega)=\min _{\underline{u}} J(\omega, \underline{u})=J\left(\omega, \underline{u}^{*}\right)
$$

The estimate $\hat{\mathrm{x}}_{\omega}$ is obtained by "reconstructing" the system state from the observed data $\underline{y}$. In accord with our optimization hypothesis, we shall assume that the data reconstructor consists of a Kalman f1lter (Ref. 22) and a least-mean squared predictor (Ref. 19) in cascade as shown in Figure $3 .^{*}$ The estimator is used to model the human's deduction of vehicle states from displayed information while the predictor models the human's compensation for his inherent time delay. The parameters, but not the structural form, of the Kalman filter depend upon the noise covariance matrices $W$ and $\underline{V}_{\omega}$ and hence upon the sampling strategy. On the other hand, it can be shown that the predictor is independent of $\omega$ and so can be determined a priori in conjunction with the optimal control gains $\underline{L}^{*}$. It is interesting to note that the Kalman filter requires for its implementation a model of the dynamic system or, put another way, a model of the environment. The connection between our data reconstruction model and Smallwood's internal model of the environment is then clear.

\footnotetext{
*It is well known that in the absence of time delay the Kalman estimator provides the best mean squared estimate of the state for the conditions of this problem. Indeed, it provides the best linear estimate under much broader conditions. Moreover, with no sampling and no observation nolse, the predictor provides the best mean squared estimate of the state when there 1s a delay (Ref.19). We have assumed that with sampling, time-delay, and observation noise of the type described, the estimation and prediction processes can be separated and further, that the tandem combination provides for a given sampling strategy the best mean squared estimate of the instantaneous state. It may be possible to prove this rigorously but we have yet to do so. At the least, such a structure should yield a good "sub-optimal" estimator.
} 
Sampler--The one remaining element in our pilot model is the sampler. It should be absolutely clear at the outset that we are referring to instrument sampling and not signal sampling. By a sampling strategy we mean a method of deciding which instrument to look at directly (foveally) at different time instances. All signals are continuously processed, but, in general, the signal on the "sampled" instrument has a lower noise level associated with it's reading.

The determination of the human operator's sampling strategy will, as with other portions of our model, rest on the assumption that the operator behaves in an approximately optimal fashion and samples his instruments accordingly (for example, to minimize estimation error). In other words, the human chooses a sampling strategy $\omega^{*}$ which minimizes $J^{*}(\omega)$ over the class of admissable strategies, 1.e.,

$$
\omega^{*}=\arg \min J^{*}(\omega)
$$

It is important to note that $\omega^{*}$ will, in general, depend on the control requirements. This will become clear in the next chapter where a precise formulation of the optimal sampling problem is given.

In order to simplify the mathematical problems associated with determining $\omega^{*}$ in the above manner, it will be assumed that the sampling strategy is independent of the time delay and, therefore, we can solve for $\omega^{*}$ by considering $\tau=0$. Heuristically, this approach seems reasonable. First of all, in the situation of pure instrument monitoring, the effect of the time delay upon sampling behavior is minimal at best and a sampling model can be constructed which ignores this delay (as in Senders, Elkind, etc.). Secondly, the prediction process associated with the time delay 
is independent of $w$ and depends only on the system parameters $A$, B, $\underline{L}^{*}$. If the converse were true then, Indeed, we need only consider the case $\tau=0$. While this conclusion must be verified mathematically, 1t should be possible to at least show that the effect of time delay on sampling behavior is of secondary importance and may be neglected.

Summary--A model structure for representing the human operator in a multivariable control task has been postulated. The model contains elements for describing the operator's inherent limitations as well as his instrument monitoring, data reconstruction and control behavior. The inherent limitations of the human operator have been modelled by lumping them into an equivalent perceptual delay and an equivalent observational noise. The noise covarlance depends on "where" the operator looks and hence influences the sampling strategy. The remainder of the model rests on the assumption that the human operator behaves optimally with respect to performing his various tasks. His control behavior is assumed to be that of an 1deal feedback controller with gains selected so as to minimize a given quadratic cost functional. The control gains are independent of the data reconstruction and instrument sampling processes, although the control input itself does depend on these processes. The data reconstruction process is modelled by a Kalman fllter and a least-mean square predictor in cascade. - The structure of the data reconstructor is fixed but the parameters depend upon the operator's sampling behavior.

The operator's sampling strategy is determined by solving an optimization problem also; in particular, by minimizing (for the case $\tau=0$ ) the functional $J^{*}(\omega)$ (see $\mathrm{Eq}$. (5)). The strategy thus obtained allows specification of the unknown parameters in the data reconstruction sub-model, thereby completing the overall model of the pilot-vehicle-display system. 
Although the details of the sampling model have yet to be specified, several noteworthy points are already apparent. First, the sampling model is such that the human's monitoring behavior depends upon the control requirements and the control actions in an explicit way. Second, the ability to process information from the peripheral visual field is also included. Finally, there are no apparent restrictions with regard to coupling of signals on various instruments. 


\section{OPTIMAL SAMPLING PROBLEM}

In the last chapter we developed the structure for a model of the human operator in a multivariable control and monitoring task. We saw that, given the optimization assumption, we would have to solve an optimal control problem in order to predict the human operator's characteristics. Here, we shall make the opt1mization problem more precise and discuss some of 1 ts implications.

The optimization problem itself is two-fold in nature. Control is avallable over not only the plant but also over the manner in which information concerning the system state is obtained and processed. Problems of this type are embedded in a more general class in which one is faced with an auxiliary optimization task within an optimal regulator control framework. The often studied problem of choosing sampling times and control input which minimize a quadratic cost for a linear discrete system (Refs. 23,24) is an example (although not applicable to the present study) of this class. The design of optimal modulating signals for optimal linear estimation, studied by Athans and Schweppe (Ref.25), is also representative of this general class of problems.

In the present study of sampling behavior we have formulated a problem in which the auxiliary optimization task relates to the measurement of the system state. Research pertinent to this problem, for the case of discrete linear regulator systems with finite terminal time, has been conducted by Kushner (Ref. 27) and by Meier, et al (Ref. 26). Kushner considered the optimum timing of state observations, when the number of possible measurements is limited. Meier, et al, considered the general problem of "controlling" a measurement subsystem. (Such problems are referred

\footnotetext{
* It will be shown that the problems of finding, optimal modulations and finding optimal sampling strategies have several aspects in common.
} 
to as "measurement-adaptive" problems, and include among others, the optimum timing problem and the problem of finding the optimum sequencing of information over a time-shared communication channel.) A significant aspect of their formulation is the inclusion of an additional term in the cost functional, representing the cost of making measurements. " They have shown that the optimal choice of a measurement control can be determined, a priori, by solving, a nonlinear matrix optimization problem of the type considered earlier by Athans and Schweppe.

The work of Meier, et al is most closely related to our method for determining human sampling behavior, and we borrow heavily from their ideas. We begin by extending their development, which was for discrete problems, to the continuous time case.

Mathematical Formulation--In this section we shall rely heavily on combined optimization and estimation theory for continuous systems (Ref. 22) in order to extend the results of Meier,et al (Ref. 26) to the continuous time case. We shall first consider finite terminal time and then extend the results to the infinite time case. In this manner we shall reduce the sampling problem to a deterministic nonlinear (matrix) optimization problem.

In order to clarify the ensuing developments we shall elaborate further on the nature of the class of sampling strategies $\Omega$. In particular, we shall characterize sampling behavior as a function of time by making $\Omega$ a function space whose elements w carry the time interval $[0, \infty)$ into a given set $\hat{\Omega}$. The set $\hat{\Omega}$ is the range space for functions $\omega(\cdot) \varepsilon \Omega$. It is the set of values assumed

* With the introduction of such a term 1t becomes posible to consider for example, constraints on scanning behavior which arise from human physiological and/or psychological factors. 
by $\omega(t)$ for $t \varepsilon[0, \infty) .^{*}$ Symbolically this is written as

$$
\omega(\cdot) \varepsilon \Omega:[0, \infty)+\hat{\Omega}
$$

or,

$$
\omega(t) \varepsilon \hat{\Omega} \quad \text { for all } t \varepsilon[0, \infty) \text {. }
$$

In this manner, choosing $\omega(\cdot) \varepsilon \Omega$ specifies the sampling behavior as a function of time and, in turn, the time variation of the noise covariance matrix $\underline{V}_{\omega}=\underline{V}(\underline{\omega}(t))$.

We shall now derive an explicit expression for $J^{*}(\omega)$, (see Eq. (5)). Letw( $) \varepsilon \Omega$ be a fixed sampling strategy, let $t_{f}$ be finite and, without loss of generality, let the matrix $\underline{R}$ in expression (3) be the identity matrix. We then define

$$
J\left(\omega, \underline{u} ; t_{f}\right)=E\left\{\frac{1}{t_{f}} \int_{0}^{t}\left[\underline{x}^{\prime} \underline{Q} \underline{x}+\underline{u}^{\prime} \underline{u}+\mathcal{L}(\omega(t))\right] d t\right\}
$$

For the Innear system

$$
\left.\begin{array}{l}
\frac{d}{d t} \underline{x}(t)=\underline{A} \underline{x}(t)+\underline{B} \underline{u}(t)+\underline{w}(t) ; \operatorname{cov}[\underline{w}]=\underline{w} ; \underline{x}(0)=\underline{0} \\
\underline{y}(t)=\underline{c} \underline{x}(t)+\underline{v}_{\omega}(t) ; \quad \operatorname{cov}[\underline{v}]=\underline{v}(w(t))
\end{array}\right\}
$$

For example, if there are 3 displays one may choose $\hat{\Omega}=\{1,2,3\}$. In this case the value of $\omega(t)$ could correspond to the instrument being viewed foveally at time $t$. The set $\Omega$ consists of piecewiseconstant time functions on $[0, \infty)$. 
the control input $\underline{u}_{w}^{*}\left(t ; t_{f}\right)$ which minimizes $(7)$ is given by

$$
\underline{u}_{\omega}^{*}\left(t ; t_{f}\right)=-\underline{L}^{*}(t) \underline{\hat{x}}_{\omega}(t)=-\underline{B}^{\prime} \underline{K}\left(t ; t_{f}\right) \underline{\hat{x}}_{\omega}(t)
$$

where $\underline{K}\left(t ; t_{f}\right)$ is the solution of the Riccati equation

$$
\underline{\dot{K}}(t)=-\underline{K}(t) \underline{A}-\underline{A}^{\prime} \underline{K}(t)-\underline{Q}+\underline{K}(t) \underline{B} \underline{B}^{\prime} \underline{K}(t)
$$

satisfying $\underline{K}\left(t_{f} ; t_{f}\right)=\underline{0}$. The term $\underline{\hat{x}}_{\omega}(t)$ is the least mean square estimate of $\underline{x}(t)$ and is generated by the dynamical system (the Kalman filter):

$$
\begin{aligned}
\frac{d}{d t} \underline{\hat{x}}(t)=\left[\underline{A}-\underline{\Sigma}_{\omega}(t) \underline{C} \underline{V}^{-1}(\omega(t)) \underline{C}\right] \underline{\hat{x}}(t)-\underline{B} \underline{u}_{\omega}^{*}\left(t ; t \underline{f}^{\prime}\right) \\
\\
+\underline{\Sigma}_{\omega}(t) \underline{C}^{\prime} \underline{V}^{-1}(\omega(t)) \underline{y}(t)
\end{aligned}
$$

where the matrix $\sum_{-\omega}(t)$ is the "error" covariance matrix

$$
\underline{\Sigma}_{\omega}(t)=E\left\{\left[\underline{x}(t)-\underline{\hat{x}}_{\omega}(t)\right]\left[\underline{x}(t)-\underline{\hat{x}}_{\omega}(t)\right]^{\prime}\right\}
$$

and satisfies the equation

$$
\frac{\mathrm{d}}{\mathrm{dt}} \underline{\Sigma}(t)=\underline{A} \underline{\Sigma}(t)+\underline{\Sigma}(t) \underline{A}^{\prime}+\underline{W}-\underline{\Sigma}(t) \underline{C^{\prime}} \underline{\mathrm{V}}_{\omega}^{-1} \underline{\mathrm{C}} \underline{\Sigma}(t)
$$

with $\underline{\Sigma}(0)=\underline{0}$.

Finally, the minimum cost

$$
J^{*}\left(\omega ; t_{f}\right)=J\left(\omega, \underline{u}_{\omega}^{*} ; t_{f}\right)
$$


is given by

$J^{*}\left(\omega ; t_{f}\right)=\frac{I}{t_{f}} \int_{0}^{t}\left\{\operatorname{tr}\left[\underline{\Sigma}_{\omega}(t) \underline{C}^{\prime} \underline{V}^{-1}(\omega(t)) \underline{c \Sigma}_{\omega}(t) \cdot \underline{K}\left(t ; t_{f}\right)+\underline{Q E}_{\omega}(t)\right]+\mathcal{L}(\omega, t)\right\} d t$

or, as an alternative expression,

$J *\left(\omega ; t_{f}\right)=\frac{1}{t_{f}} \int_{0}^{t}\left\{t r\left[\underline{W} \underline{K}\left(t ; t_{f}\right)+\underline{L}^{*}(t) \underline{L}^{*}(t) \underline{\Sigma} \omega(t)\right]+\mathcal{L}(\omega, t)\right\} d t(I 6)$

In Eq. (16) the first term represents the cost resulting, from the driving noise $\underline{w}(t)$ and is independent of $\omega$. The second term represents the degradation in cost arising from the estimation noise $\underline{V}_{\omega}$. The third term represents the "sampling cost" as discussed earlier.

In the above development it is noteworthy that:

1) $L^{*}(t)$ is independent of the noise processes, and therefore independent of $w$. (This is the wellknown separability result for linear systems.) However, the converse is not true as $\omega$ depends on $L^{*}$ through the second term of Eq. (I6).

2) $\sum_{\omega}(t)$ is independent of the terminal time $t_{f}$ and

We now extend the above results to the case $t_{f}+\infty$. We assume (although it may be possible to show rigorously) that

$$
\lim _{f^{+\infty}}\left\{\min _{\underline{u}} J\left(\omega ; \underline{u} ; t_{f}\right)\right\}=\min _{u} J(\omega ; \underline{u}, \infty)=J^{*}(\omega)
$$


and that this minimum is achieved at

$$
\underline{u}^{*}(\omega)=\lim _{t_{f} \rightarrow \infty} \underline{u}^{*}\left(\omega ; t_{f}\right)
$$

The left-hand limit of Eq. (17) is readily shown to exist. Since

$$
\lim _{t_{f \rightarrow \infty}} K\left(t ; t_{f}\right)=\underline{\bar{K}}=\text { constant }
$$

where $\bar{K}$ is the unique positive definite solution of

$$
\underline{O}=\underline{K} \underline{A}+\underline{A}^{\prime} \underline{K}+\underline{Q}-\underline{K} \underline{B} \underline{B}^{\prime} \underline{K}
$$

we have

$$
\lim _{t \rightarrow \infty} L^{*}(t)=\underline{L}^{*}=-\underline{B}^{\prime} \bar{K}
$$

Furthermore, since $\Sigma_{\omega}(t)$ is independent of $t_{f}$ we obtain

$$
\begin{aligned}
\lim _{t_{f \rightarrow \infty}} J^{*}\left(\omega ; t_{f}\right) & =\operatorname{tr}[\underline{W} \underline{\bar{K}}]+\operatorname{tr}\left[\underline{L}^{*} \underline{\Sigma}_{\operatorname{avg}}(\omega) \underline{L} \underline{H}^{\prime}\right]+\mathcal{L}_{\mathrm{avg}}(\omega) \\
& =J^{*}(\omega)
\end{aligned}
$$

where

$$
\begin{aligned}
& \underline{\Sigma}_{\operatorname{avg}}(\omega)=\lim _{f^{\rightarrow \infty}} \frac{I}{t_{f}} \int_{0}^{t_{f}} \underline{\Sigma}(w, t) d t \\
& \mathcal{L}_{\text {avg }}(\omega)=\lim _{t_{f} \rightarrow \infty} \frac{1}{t_{f}} \int_{0}^{f} L(w, t) d t
\end{aligned}
$$


are the time-average values of $\underline{\Sigma}(\omega, t)$ and $(\omega, t)$ over the infinite interval $[0, \infty)$.

In addition, the limit (18) becomes

$$
\underline{u}^{*}(\omega)=-\underline{L}^{*} \hat{\underline{x}}_{\omega}(t)
$$

where $\hat{x}_{w}(t)$ is generated by Eq. (II) with $\underline{u}_{\omega}^{*}\left(t ; t_{f}\right)$ replaced by $-\underline{L}^{*} \hat{\mathrm{x}}_{\omega}(t)$.

The element $\omega^{*}(\cdot) \varepsilon \Omega$ (or equivalently $\omega^{*}(t) \varepsilon \hat{\Omega}$ for $t \varepsilon[0, \infty)$ ) which minimizes $J^{*}(\omega)$ will be used to predict the pilot's scanning behavior. This is in accordance with our assumption that the pllot acts in a (near) optimal fashion and thereby chooses an optimal sampling pattern. It remains, therefore, to minimize $J^{*}(\omega)$ over $\Omega$, or equivalently, since the first term in $J^{*}(\omega)$ is independent of $\omega$, to minimize

$$
I(\omega)=\operatorname{tr}\left[\underline{L}^{*} \underline{\operatorname{avg}}(\omega) \underline{L}^{*}\right]+\mathscr{L}_{\mathrm{avg}}(\omega)
$$

The mathematical optimization problem of finding $\omega *$ is exceedingly difficult at this stage because of the form of the dependence of $I(\omega)$ upon $\omega$. For example, if $\omega_{1}(\cdot)$ and $\omega_{2}(\cdot)$ are two elements of $\Omega$ which differ only over a finite interval $[a, b] c[0, \infty)$ then

$$
I\left(\omega_{1}\right)=I\left(w_{2}\right)
$$

This implies that there is no unique element $\omega^{*}(\cdot) \in \Omega$ which minimizes $I(\omega)$. Thus, the application of variational techniques to * In fact there are an infinity of elements in $\Omega$ which minimize $I(\omega)$. 
solve the problem in its present form is virtually useless, and it becomes necessary to introduce further restrictions on the class $\Omega$.

The assumption we shall make 1 s that any element $\omega(\cdot) \varepsilon \Omega$ is periodic with period $T<\infty$, where $T$ is fixed but arbitrary. Hence,

$$
\omega(t)=\omega(t+T) \quad \text { for all } t \varepsilon[0, \infty)
$$

The mathematical assumption (25) is tantamount to the physical assumption that the pilot's sampling behavior is periodic with period $\mathrm{T}$.

With the assumption (25) we have, in turn, that $\underline{V}(\omega(t))$ is also periodic with period $T$. It is then possible to show:

1) There exists a unique periodic solution of the variance equation (13) having period $T$. Call this solution $\underline{\underline{\Sigma}}(\omega, t)$.

2) For any positive semi-definite matrix $F$ let $\Sigma_{F}(\omega, t)$ be the solution of $\mathrm{Eq}$. (13) satisfying $\underline{\Sigma}_{F}(\omega, 0)=\underline{F}$, then

$$
\lim _{t \rightarrow \infty} \underline{\Sigma}_{F}(w, t)=\underline{\Sigma}(\omega, t)
$$

The above two statements imply that

$$
I(\omega)=\frac{1}{T} \operatorname{tr}\left\{L^{*} \int_{0}^{T} \underline{\Sigma}(\omega, t) d t \underline{L}^{*}\right\}+\frac{1}{T} \int_{0}^{T} \mathcal{L}(\omega, t) d t
$$

Consequently, the determination of $\omega^{*}$ has been reduced to the following nonlinear optimization problem: 
Optimal Sampling Problem: Given $T<\infty$. Find the element $\omega * \varepsilon \Omega$ which minimizes $I(\omega)$, where $\underline{\Sigma}(\omega, t)$ satisfies

$$
\frac{d}{d t} \underline{\Sigma}(t)=\underline{A}^{\prime} \underline{\Sigma}(t)+\underline{\Sigma}(t) \underline{A}+\underline{W}-\underline{\Sigma}(t) \underline{C}^{\prime} \underline{V}^{-1}(\omega(t)) \underline{C \Sigma}(t)(28)
$$

with the boundary condition

$$
\underline{\Sigma}(0)=\underline{\Sigma}(\mathrm{T})>\underline{0} .
$$

Thus far, the sampling period $T$ has been considered a flxed but arbitrary parameter. However, in order to completely specify the sampling behavior of the human operator, we must also specify $T$. This is accomplished, in accordance with our optimization hypothesis, in the following manner. For a particular value of $T$ the optimal sampling problem can, in principle, be solved for $\omega^{*}$ and $I\left(\omega^{*}\right)$. Thus, $\omega^{*}(\cdot)$ will parametrically depend on $T$ and we write

$$
\left.\begin{array}{l}
\omega_{\mathrm{T}}^{*}(\cdot) \equiv \omega^{*}(\cdot) \\
I\left(\omega_{\mathrm{T}}^{*}\right) \equiv I\left(\omega^{*}\right)
\end{array}\right\}
$$

We now vary $T$, and for each $T$ compute $\omega_{T}^{*}(\cdot)$ as well as $I\left(\omega_{T}^{*}\right)$. Thus, $I\left(\omega_{\mathrm{T}}^{*}\right) \cdot$ will be a scalar function of $\mathrm{T}$ and we let

$$
T^{*}=\arg \min _{T} I\left(\omega_{T}^{*}\right)
$$

be the requisite cholce of scan period (1.e., the optimal scan period). In this manner, $\omega_{\text {T* }}^{*}$ will describe the optimal sampling strategy. 
In order to accomplish the minimization indicated in Eq. (31), It will be necessary to introduce certain nonrepetitive assumptions and the class $\Omega$. As $T$ increases, the set $\Omega$ will encompass a wider class of sampling strategies and not merely repetitive cycles of those sampling strategies contalned in a set $\Omega$ for smaller values of $T$. (Recall that a function which is periodic in $T$ is also periodic in $2 \mathrm{~T}$.

Solving the Optimal Sampling Problem--The deterministic optimization problem derived above is of a special form. The error covariance matrix $\Sigma(t)$ may be interpreted as a system state, the matrix Riccati equation plays the role of a dynamic system and the function $\omega(t)$ is regarded as a control input. Such a nonlinear optimization problem will normally have to be solved computationally. Several approaches, based on different characterizations of the optimal solution, are possible.

Athans and Schweppe (Ref. 25) used a matrix version of the Maximum Principle in their investigation of a nonlinear optimization problem of this type. They considered the problem of minimizing

$$
I(\omega)=\operatorname{tr} \underline{\Sigma}(T)
$$

subject to the boundary conditions $\underline{\Sigma}(0)=\underline{\Sigma}_{0}=$ given; the control, $\omega(t)$, was constrained. Using the (matrix) Maximum Principle, they obtained necessary conditions for optimality. Such conditions can serve as the basis for varlous computing algorithms. However, since our cost functional is of a different form and since the constraints on $\omega$ will not be local in nature if we constrain the sampling rates, the techniques of Ref. 25 would have to be extended before they could be applied to the problem of determining $\omega^{*}$. In addition to using the Maximum Principle to compute $\omega^{*}$, there also 
exists the possibility of applying it (for the free terminal time case) to the problem of determining the optimal sampling period.

Meier, et al (Ref. 26) used dynamic programming to solve some measurement adaptive problems. Dynamic programming methods have distinct advantages so long as one is not confronted with the "curse of dimensionality" (Ref. 28). For the optimal sampling problem, the dimensionality, from a dynamic programming stand point, is determined by the number of instruments to be sampled. Thus, in problems in which the number of instruments to be sampled is not too large, dynamic programming techniques might prove quite useful.

Although the development of theoretically based computational algorithms for solving the optimal sampling problem is a desirable, if not necessary, goal, it is possible to solve simple versions of the problem by numerical search techniques. It is always possible to calculate the cost $I\left(\omega_{1}\right)$ for a given $\omega_{1} \varepsilon \Omega$. This calculation is nontrivial because of the split boundary conditions (29) on $\underline{\Sigma}(t)$. In order to evaluate $I\left(\omega_{1}\right)$ it is first necessary to compute $\underline{\Sigma}(0)$ explicitly. Once this is accomplished, $\underline{\Sigma}(t)$ is readily obtained by integrating Eq. (28). We note that since Fq. (28) possesses a unique periodic solution (corresponding to a given periodic sampling strategy) it is possible to compute $\underline{\Sigma}(0)$ by means of the convergent algorithm

$\underline{\Sigma}_{(1+1)}(0)=\left[\underline{\Psi}_{21}(T, 0)+\underline{\Psi}_{22}(T, 0) \underline{\Sigma}_{i}(0)\right]\left[\underline{\Psi}_{11}(T, 0)+\underline{\Psi}_{12}(T, 0) \underline{\Sigma}_{i}(0)\right]^{-1}$ 
where the $2 n \times 2 n$ matrix

$$
\Psi\left(t, t_{0}\right)=\left[\begin{array}{l:l}
\Psi_{11}\left(t, t_{0}\right) & \Psi_{12}\left(t, t_{0}\right) \\
\hdashline \Psi_{21}\left(t, t_{0}\right) & \Psi_{22}\left(t, t_{0}\right)
\end{array}\right]
$$

is the transition matrix associated with

$$
\underline{Z}(t)=\left[\begin{array}{c:c}
-\underline{A} & -\underline{C} \underline{V}^{-1}(\omega(t)) \underline{C} \\
\hdashline-\underline{W} & \underline{A}
\end{array}\right]
$$

i.e.,

$$
\underline{\Psi}\left(t, t_{0}\right)=\underline{Z}(t) \underline{\Psi}\left(t, t_{0}\right) ; \quad \underline{\Psi}\left(t_{0}, t_{0}\right)=I
$$

Thus, $I(\omega)$ can be evaluated for a given $\omega$ and one can numerically compare the costs $I\left(\omega_{1}\right)$ and $I\left(\omega_{2}\right)$ associated with two different sampling strategies $\omega_{1}$ and $\omega_{2}$. In this manner it becomes possible to perform a numerical search for $\omega^{*}$ for a given value of $T$. One can then repeat the procedure for various values of $T$ and thus determine the minimum of $I$ with respect to $\omega$ and $T$.

Summary and Comments--In this chapter a precise formulation of the optimum sampling problem was developed. In the course of this development several points worthy of further elaboration emerged. First, and perhaps most noteworthy, was the assumption that the sampling, was periodic. This assumption is not as restrictive as it may first seem. Within a period $T$, any arbitrary sequence of instrument selections is possible, provided that the entire sequence is not repeated. This means that each instrument may be looked at several times during a single period $T$ and that the interval between these looks is not necessarily the same. 
The assumption of periodicity is necessitated by the consideration of an infinite time problem and is tantamount to assuming that there will be some "steady state" sampling behavior. If we so choose, the optimization framework can be used to predict human sampling behavior for a control task of finite duration and thus obviate the need for the periodicity assumption. This is accomplished by minimizing $J(\underline{u}(\cdot), w)$ for a finite $t_{f}$ (as opposed to taking the limit as $\left.t_{f}+\infty\right)$. The results of such an approach will be very similar to those obtained for the infinite time case, except that $t_{f}$ replaces $T$, and the inftial condition $\Sigma(0)$ must be given in the problem statement. For this case the optimal $\omega^{*}$ will represent the scan pattern for the run duration and not averaged scanning behavior. It should be noted that if $t_{f}$ is finite the optimal control gains $L^{*}$ are no longer constant.

A second point is the possibility of introducing sampling: rate constraints in one of several different ways. Such constraints are clearly present in the human operator and, if we are to obtain meaningful results from our model, it seems l1kely that we will have to account for them in our representation. Explicit sampling rate constrains can be considered by bounding the derivative of $\omega(t)$; this artifice is similar to that suggested by Athans and Schweppe (Ref. 25) for constraining signal bandwidths. Alternatively, it may be possible to include a cost for high sampling rates in the functional $I(\omega)$ by choice of a suitable form for the term $\mathcal{L}(\omega, t)$. Sampling rates can also be constrained implicitly by Introducing certain transition or "dead" times which corresponds to physiological read-out or data acquisition times. This was the approach taken by smallwood and is also the approach we shall use in the numerical example of the next chapter.

The connection between control requirements and sampling behavior was also clarified in the above development. For the 
joint control and sampling problem the cost function $I(\omega)$ is given by (see Eq. (24))

$$
I(\omega)=\operatorname{tr}\left[L^{*} \underline{a v g}_{\left.L^{*} \cdot\right]+\mathscr{L}} \operatorname{avg}(\omega)\right.
$$

Now, if the operator's task is simply to monitor the instruments and obtain a minimum variance estimate of the system state, it can readily be shown that the optimal sampling strategy is obtained by solving for $\omega^{*}$ to minimize

$$
I(\omega)=\operatorname{tr}\left[\underline{\Sigma}_{\mathrm{avg}}\right]+\mathscr{L}_{\mathrm{avg}}(\omega)
$$

Such a cost function would assign equal weight to errors in est1mation in any of the state variables. If the operator were instructed to consider some state variable errors more important than others, he could do so by appropriately modifying the cost function, that is, by choosing I to be of the form

$$
I=\operatorname{tr}\left[\underline{M} \underline{\operatorname{avg}^{\underline{M}}} \underline{M}^{\prime}\right]+\mathscr{L} \operatorname{avg}^{(\omega)}
$$

The matrix $M$ weights the various estimation errors according to the preselected criteria. But, this is exactly the role played by the optimal gain matrix $\underline{L}^{*}$ in our formulation of the joint sampling and control problem. Thus, the sampling, strategy $\omega$ is selected to minimize a weighted estimation error where the weightIng is consistent with the contributions of the state variables to the optimal feedback law. The matrix $L^{*}$ depends only on the original weighting matrices, $\underline{Q}$ and $\underline{R}$, in the "overall" performance index. Hence, it is possible to investigate, in a syotematic way, the changes in human sampling behavior which result from different control requirements (that is, from different weightings $\underline{Q}$ and $\underline{R}$ ). 
Finally, we saw that in order to determine the sampling behavior, it was necessary to solve a nonlinear deterministic opt1mization problem. Analytic solution to the problem is highly unlikely and we are faced with the probability that solutions can only be obtained computationally. Several approaches to obtainIng an efficient computational algorithm were discussed but this remains a prime area for further research. It is sometimes feasible, however, to obtain solutions by a numerical search and this is done for a simple example in the next chapter. 


\section{A SIMPLE EXAMPLE}

In this chapter the foregoing concepts and theory are applied to a specific control and monltoring task. In order to illustrate the method and avoid cumbersome numerical calculations, we shall consider a simplified example. We shall illustrate: 1) a method of choosing $\Omega$ and the dependence of $\underline{V}(\omega)$ upon $\omega, 2$ ) a method for constraining sampling rates, and 3 ) the effects of certain parametric variations upon predicted sampling behavior.

A Two-Axis Tracking Problem--We assume that a human operator is presented with two separate displays each being driven by the output of a first order dynamical system of the form

$$
\dot{x}_{1}(t)=a_{1} x_{1}(t)+b_{1} u_{1}(t)+w_{1}(t) ; \quad \operatorname{cov}\left[w_{1}(t)\right]=w_{11}
$$

Thus, the two sets of dynamics $(1=1,2)$ are completely noninteracting. We assume that $x_{1}$ and $x_{2}$ are presented on display 1 and 2 respectively, and that the quantities viewed by the human operator will be (cf. Eq: (8))

$$
y_{1}(t)=x_{1}(t)+v_{1}(\omega) ; \quad \operatorname{cov}\left[v_{1}(\omega)\right]=v_{11}(\omega), 1=1,2
$$

The additive observation noise $v_{1}(\omega)$ is associated with display 1 and depends on the sampling behavior $\omega(t) .^{*}$ We defer, for the moment, a discussion of this dependence and of the sampling class $\Omega$.

The control task is to pick $u_{1}(t)$ and $u_{2}(t)$ so as to minimize

*It is assumed that $v_{1}(t)$ and $v_{2}(t)$ are independent. 


$$
\begin{aligned}
J\left(u_{1}, u_{2}\right)= & \lim _{t_{f} \rightarrow \infty} E\left\{\frac { 1 } { t _ { f } } \int _ { 0 } ^ { t } \left[q_{11} x_{1}^{2}(t)+q_{22} x_{2}^{2}(t)\right.\right. \\
& \left.\left.+u_{1}^{2}(t)+u_{2}^{2}(t)\right] d t\right\}
\end{aligned}
$$

Our objective is to predict the human sampling behavior associated with the given control task. We shall accomplish this by solving the optimal sampling problem for $\omega^{*}$.

Cholce of Sampling Parameters--The solution of the optimal sampling problem requires the explicit specification of the class of sampling strategies $\Omega$ and the functional relationship between $\underline{V}(\omega)$ and $\omega(t)$. For the example being considered we shall include sampling rate constraints within these specifications.

1. Sampling Class

We consider a fixed (but arbitrary) scan period, T. At any time $t \in[0, T]$ it is assumed that the pilot is either looking foveally at display 1 or display 2 , or else, is in the transitional phase (i.e., switching attention). We let $\omega(t)=1,2$ or 0 to correspond to each of the above cases, respectively. Hence, the set $\hat{\Omega}$ which is the range space of $\omega(\cdot) \varepsilon \Omega$ (see pg. (29)) is

$$
\hat{\Omega}=\{1,2,0\}
$$

and $\omega(\cdot)$ es maps $[0, T]$ into $\hat{\Omega}$.

Note that $\omega(t)$ so defined is piecewise constant, and over any time interval may be described by a sequence of values $\{1,0,2,0,1, \ldots\}$. Notice further that sequences of the form $\{\ldots, 1,2, \ldots\}$ are not allowed. We assume that every transition of foveal attention between displays requires a finite transition or "dead" time, $t_{0}$. 
Examination of some of our pilot eye movement data indicates that a reasonable value of $t_{0}$ is 0.1 sec. (This was also the value Smallwood assumed.)

We are interested in a sequence in which the pilot samples each instrument once per scan period. Thus, the sequences of values of $\omega(t)$ for any $\omega(\cdot) \varepsilon \Omega$ is (assuming for convenience, that instrument 1 is sampled first) $\{1,0,2,0\}$. If we let $t_{1}$ and $t_{2}$ be the times that a pilot spends in viewing instrument 1 and 2 , respectively, we have

$$
T=t_{1}+t_{2}+2 t_{0}
$$

Thus, any element we can be characterized by the single number $t_{1}$ (or $t_{2}$ ) since $T$ and $t_{0}$ are given. In summary, then, any element $\omega(\cdot) \varepsilon$ is piecewise constant over $[0, \mathrm{~T}]$ and takes on the sequence of values $\{1,0,2,0\}$.

2. Characterization of $\underline{V}(\omega)$

Since $\omega(t)$ can take on only one of three values, $(0,1$ or 2) $\underline{V}(\omega)$ may be characterized by three matrices $\underline{V}(1), \underline{V}(2)$, $\underline{V}(0)$. The matrices $\underline{V}(1)$ and $\underline{V}(2)$ correspond, respectively, to the observation noise covariances for foveal viewing of display 1 or display 2, while $\underline{V}(0)$ is the corresponding covariance matrix for switching attention. Since we have assumed independent noise processes, the above matrices are diagonal. Hence, $\underline{V}(\omega)=\operatorname{diag}\left[v_{11}(\omega), v_{22}(\omega)\right]$.

Here, we shall assume that the elements of $\underline{V}(1)$ and $\underline{V}(2)$ are given quantities in the problem formulation. These parameters relate to, and are identified with, the display system and 1 ts interaction with the human operator. For instance, the numbers $v_{11}(1)$ and $v_{22}(2)$ will be less than $v_{11}(2)$ and $v_{22}(1)$, respectively, 
since foveal viewing of a given display will result in less observational noise than will peripheral viewing. Similarly, if the display separation is increased (or decreased) we might expect the peripheral noise covariances $v_{11}(2)$ and $v_{22}(1)$ to similarly increase (or decrease).

The elements of $\underline{V}(0)$ will be relatively large since we assume that no display is seen accurately during the transition between instruments. An alternative assumption would be to consider that no instrument is viewed foveally but that some (in this case both) are viewed peripherally during a transition. This latter assumption would also result in relatively large values for the elements of $\underline{V}(0)$. Examination of the variance equation (28) shows that as the elements of $\underline{V}$ increase, the solution, $\underline{\Sigma}$ becomes increasingly independent of the magnitudes of these elements. This implies that a fairly gross approximation to $\underline{V}(0)$ may be adequate for predicting sampling behavior. If such is the case it would be fortuitous since accurate determination of the elements of $\underline{V}(0)$ seems an extremely difficult task. Indeed, determining the required values of $\underline{V}(1)$ and $\underline{V}(2)$ is also nontrivial. However, for our present purposes, we may avoid these difficulties by making a priori selection for the values of the various noise covariances.

Optimal Sampling Problem for Two-Axis Tracking--We are now in a position to solve the optimum sampling problem. We are given the system dynamics, cost criterion, class of sampling strategies and observation noise covariances. It is then easy to show (since display 1 and 2 are independent) that the optimal sampling problem reduces to:

Given $T<\infty$, find the element $\omega^{*} \varepsilon \Omega$ which minimizes

$$
I(\omega)=\frac{I}{T}\left\{l_{I}^{2} \int_{0}^{T} \sigma_{11}(t) d t+\ell_{2}^{2} \int_{0}^{T} \sigma_{22}(t) d t\right\}
$$


where

$$
\begin{aligned}
& \dot{\sigma}_{11}(t)=2 a_{1} \sigma_{11}-w_{11}+\sigma_{11}^{2}(t) / v_{11}(\omega(t)) \\
& \dot{\sigma}_{22}(t)=2 a_{2} \sigma_{22}(t)-w_{22}+\sigma_{22}^{2}(t) / v_{22}(\omega(t)) \\
& \sigma_{11}(0)=\sigma_{11}(T)>0 \\
& \sigma_{22}(0)=\sigma_{22}(T)>0 \\
& \ell_{1}=\left(a_{1}+\sqrt{a_{1}^{2}+q_{1} b_{1}^{2}}\right) / b_{1} \\
& l_{2}=\left(a_{2}+\sqrt{a_{2}^{2}+q_{2} b_{2}^{2}}\right) / b_{2}
\end{aligned}
$$

Since $\omega(t)$ assumes the values 1,2 or 0 in the sequence $\{1,0,2,0\}$ and since $T$ and $t_{0}$ are fixed, the determination of $\omega^{*}(\cdot)$ is equivalent to the determination of $t_{I}$. Thus, $I(\omega)$ is a function of the single parameter $t_{1}$ and the value of $t_{1}$ which minimizes $I(\omega)$ can be obtained by a simple numerlcal search. For a fixed value of $t_{1}$ the value of $I(\omega)$ is obtained by first solving Eq. (43) for $\sigma_{11}(0)$ and $\sigma_{22}(0)$. This is readily accomplished since $v_{11}(t)$ and $v_{22}(t)$ are merely plecewise constant. We then integrate Eqs. (42) and (4I) to obtain $I(\omega)$.

Thus, for a fixed value of $T$ we can obtain $\omega^{*}(\cdot)$. In order to obtain the optimum scan period $T$ it is necessary to investigate the behavior of $I\left(\omega^{*}\right)$ as a function of $T$. The value of $T$ which minimizes $I\left(\omega^{*}\right)$ is denoted by $T^{*}$ and serves as a prediction of human scan per1od. By the nature of the class $\Omega$, $T^{*}$ must necessarily be greater than $2 t_{0}$ (except for the case when foveal 
attention is directed entirely at one display). Finally, the sampling function $w^{*}$ which corresponds to $T^{*}$ will serve to predict mean dwell times for instrument $I$ and 2 .

For numerical convenience it will be assumed that $v_{11}(0)=v_{11}(2)$ and $v_{22}(0)=v_{22}(1)$. This corresponds to peripheral viewing of both displays during a transition between instruments. This assumption is not expected to appreciably affect sampling behavior since $v_{11}(2)$ and $v_{22}(1)$ will be greater than $v_{11}(1)$ or $v_{22}(2)$ (see discussion on $\mathrm{pg}$. (46)).

Results and Discussion--In this section we shall examine some of the two-axis sampling behavior predictions of our optimal sampling model. We first examine a "nominal" case and determine the optimal sampling period and the average dwell-times for each instrument. Then we examine the effects of various system changes on the predicted sampling behavior. We shall investigate system changes of the following types:

1. Changes in controlled element dynamics.

2. Changes in noise covariances.

3. Changes in cost functional weightings.

The Nominal Case--We choose for a nominal case the situation in which both axes are identical, $1 . e$. , the dynamics, the driving nolse covariances, the associated observation nolse matrices, and the cost functional weightings are the same for each axis. The nominal controlled element dynamics correspond to the case $a_{1}=a_{2}=0$ (this is equivalent to the $\mathrm{k} / \mathrm{s}$ dynamics, with $\mathrm{k}=1$, frequently used in tracking experiments). The remaining parameters are chosen arbitrarliy to be 


$$
\begin{aligned}
\mathrm{b}_{1}=\mathrm{b}_{2}=1 ; \mathrm{q}_{1}=\mathrm{q}_{2}=10 \\
\underline{\mathrm{V}}(1)=\left[\begin{array}{cc}
05 & 0 \\
0 & 1.0
\end{array}\right] ; \underline{\mathrm{V}}(2)=\left[\begin{array}{cc}
1.0 & 0 \\
0 & .05
\end{array}\right] ; \underline{\mathrm{V}}(0)=\left[\begin{array}{cc}
1.0 & 0 \\
0 & 1.0
\end{array}\right] \\
\underline{\mathrm{W}}=\left[\begin{array}{cc}
2 & 0 \\
0 & .2
\end{array}\right]
\end{aligned}
$$

A transition time $t_{0}$ of. I seconds is assumed throughout.

The results for the nominal case are shown as the circled points in Figures 4-12. We see that the optimum sampling model predicts, as one would expect, that an equal time is spent on each display. Note, however, that this is not $50 \%$ of the sampling period since 2/loths of a second is lost in the switching of attention. The optimal sampling period $T^{*}$ is found to be approximately 1.1 seconds. The cost due to sampling $I(\omega)$ 1s approximately 2.8 . No slgnificance can be attached to the numerical values of $\mathrm{T}^{*}$ and $I(w)$ at this time since we presently have no experimental data corresponding to an equivalent situation. Levison and Elkind (Ref. 16) did look at a two-axis tracking task with $\mathrm{k} / \mathrm{s}$ dynamics on each axis. They found that when the inputs to both axes were the same the operator spent about equal time viewing each axis. They obtalned average sampling periods in the nelghborhood of 2.5 to 3 seconds. However, they used relatively low bandwidths inputs and, hence, there is no reasonable basis for comparing their results with those obtained here.

Effects of Controlled Element Dynamics--In this section we examine the effects of varying $a_{1}$ and $a_{2}$, that is, the controlled element dynamics, while holding the remaining parameters fixed. The results of such variations are shown in Figures 4-6. The results agree well with intultion. From Figures $4 \mathrm{a}$ and $4 \mathrm{~b}$ we see 
that a larger percentage of time is spent viewing the axis which is more difficult to track, that is, the axis with the more unstable dynamics. Indeed, as one axis becomes more stable with respect to the other, one soon reaches the point where the best thing to do is to spend all of the time looking foveally at the more unstable axis; the more stable axis is then tracked solely on the basis of information obtained from peripheral viewing of the associated display.

In Figure 5 we see the effects of changes in the controlled element dynamics on the sampling period. Specifically, we have plotted the minimum sampling period $T^{*}$ as a function of $a_{2}$ for various fixed values of $a_{1}$. This family of curves has several interesting, and perhaps important, properties. First, for $a_{1}$ fixed, the optimal sampling period $T^{*}$ has a unique minimum when considered as a function of $a_{2}$ and this minimum occurs, at least numerically, when $a_{2}$ is equal to $a_{1}$. Moreover, the locus of these minima, $1 . e .$, the envelope of the family of curves, seems to be a well-defined, smooth curve. We hasten to point out that we presently have no analytic results which would support drawing general conclusions from these findings. However, it is difficult to believe that the numerical results are purely coincidental. Another interesting point concerning these curves is that $T^{*}$ has a sharper minimum for values of $a_{1}$ corresponding to more stable dynamics. The value of this minimum, however, decreases as the systems become more unstable. The decreasing minimum sampling period can be explained by noting that as both systems become more and more unstable it becomes necessary to view each display foveally more often in order to avoid large estimation errors (associated with peripheral viewing). The sharpness of the minima

Recall that scanning frequency is inversely proportional to $T^{*}$. 
is more difficult to explain. It may be related to the decreasing value of the minimal optimal sampling perlod; thus, as Min $\mathrm{T}^{*} \mathrm{de-}$ creases, that is, as the systems become more unstable, the relative contribution of the dead time $2 t_{0}$ to the sampling period increases and, consequently, small variations in the dynamics of one system do not appreciably alter the optimal sampling period.

Finally, the effects of changes in controlled element dynamics on the cost for sampling is illustrated in Figure 6. As expected the costs increase as the systems become more unstable.

Effects of Observational Noise Covariances--As noted earlier, the observation noise matrix will be used to account for the effects of imprecise measurements. This lack of precision may result from display related phenomena or inherent pilot limitations. Quantitative specification of the observation noise is, however, an extremely difficult task. Here, we content ourselves with an examination of the changes in sampling behavior which result from variations in both foveally and peripheral nolse levels, as well as in the ratio of the two. All other system parameters are fixed at their nominal levels for this investigation.

In Figures 7 and 8 we show the effects of changes in peripheral and foveal noise levels on the optimal sampling period and the sampling cost, respectively. Several conclusions may be drawn from examination of these figures. First, for fixed values of peripheral noise, increases in the foveal noise result in increases in the sampling period. This may be explained by the fact that as the foveal nolse level approaches that of the peripheral noise, the quality of information obtained by foveal viewing degrades to the point where it is not much better than that which can be obtained by peripheral viewing. Consequently, there is less and less reason to switch foveal attention. In the limit, when the two noise levels are equal, there is no reason to switch foveal attention and one would expect the sampling period to approach infinity. 
The curves of Figure 7 do indeed show very rapid increases in the sampling period as the foveal nolse level approaches the peripheral noise level. On the other hand, for fixed foveal noise levels we find that as the peripheral noise increases, the sampling period decreases and rapidly tends to some nearly constant value. The fact that for large peripheral noise levels, small changes in those noise levels do not appreciably affect the sampling period, is not surprising and was discussed earlier. As peripheral noise levels Increase the amount of information which can be obtained from peripheral viewing decreases. Beyond a certain point, the only useful information is obtained during foveal viewing and further increases in peripheral noise levels have negligible effect on sampling behavior.

An appealing interpretation of the above results is possible. The foveal noise level can be considered to be a function of the display design. High foveal noise levels correspond to a poor display. Thus, if the foveal noise is high, longer reading times and, consequently, longer sampling periods are required. The ratio of foveal noise to perlpheral nolse, for a fixed foveal noise level, can be interpreted as a measure of display separation. As the displays are separated this ratio decreases or what is the same, for fixed foveal noise, the peripheral noise increases. Thus, one reaches a point of display separation beyond which increases in the separation do not appreciably affect the sampling period since nearly all useful information must be obtained from foveal viewing. Conversely, as the foveal to peripheral noise ratio increases, the displays are moved closer together and the necessity for visual sampling decreases. When the ratio is one, the instruments may be considered superimposed or integrated. Then, there is no necessity for visual sampling and the sampling period approaches infin1 ty. 
Finally, the cost $I(\omega)$ increases monotonically with increases in elther foveal or peripheral noise. The costs also tend to level off as the perlpheral noise increases so that the sensitivity of the cost to changes in peripheral noise level for large peripheral noise levels is small. These results are consistent with our expectations.

Effects of Input Noise Covariance--The effects of changes in input noise levels, for fixed observation noise, on sampling behavior are illustrated in Figures 9 and 10. Figure 9 shows that as the input noise level increases, the sampling period $T^{*}$ decreases. Increasing the input noise covariance may be interpreted as making the task more difficult. This, as in other instances, results in more frequent sampling. As expected, the effect is simflar to that obtained when the input nolse level is fixed and the observation nolse is decreased. It would appear that the significant parameter is the ratio of input and observation noise covariances.

In Figure 10, we see the effects at having different input nolse levels on each axis. In particular, we have plotted the percent time spent on instrument 2 vs. the driving noise level on axis $2\left(w_{22}\right)$ for various values of axis 1 input nolse levels $\left(w_{11}\right)$. We find that for fixed values of $w_{11}$ the percent time spent on instrument 2 increases as $w_{22}$ increases. This apparently results from the fact that the control task becomes more difficult as the input noise level increases.

Effects of Control Requirements--One of the more significant aspects of the optimal sampling model is the interaction between control requirements and sampling behavior. Here, we briefly examine the effects of changes in control requirements on sampling behavior for the two axis tracking problem. 
In the optimization framework we are using, control requirements are explicitly stated in terms of a selection for the weighting matrices $\underline{Q}$ and $\underline{R}$. We have "normalized" this selection by letting $R$ be the identity matrix. Thus, for the two axis tracking problem we need only choose $q_{1}$ and $q_{2}$ (slnce $\underline{Q}$ is assumed diagonal). The most significant quantity is actually the ratio of these two weighting coefficients for this simple case.

Figures 11 and 12 show the effects of changing the ratio $q_{2} / q_{1}$, for fixed $q_{1}$, on the fraction at time spent on instrument 2 and the optimal sampling period, respectively. The results are as expected. Figure 11 shows that as $\mathrm{q}_{2}$ decreases, i.e., as control of $x_{2}$ becomes less important, the time spent on instrument 2 also decreases, in spite of the fact that the two axes are identical in all other respects. It is interesting to note that the point at which instrument 2 is not looked at, at all, occurs prior to $q_{2}=0$. This is apparently due to the inclusion of a cost for switching attention $\left(2 t_{0}\right)$. Thus, as $q_{2}$ decreases, a point is reached where the "penalty" for switching attention exceeds the benefits of obtaining better information, by foveal viewing, concerning $x_{2}$.

From Figure 12, we see that the optimal sampling period $T^{*}$ is a minimum when $q_{2} / q_{1}=1$, i.e., when both states are equally weighted. In addition, small departures from equal weighting do not materially increase the sampling period. As $q_{2} / q_{1}$ becomes elther very small or very large the sampling period increases rapidly. This is to be expected since both cases correspond to a situation in which one instrument is not very important and, hence, there is little need to look at it. 


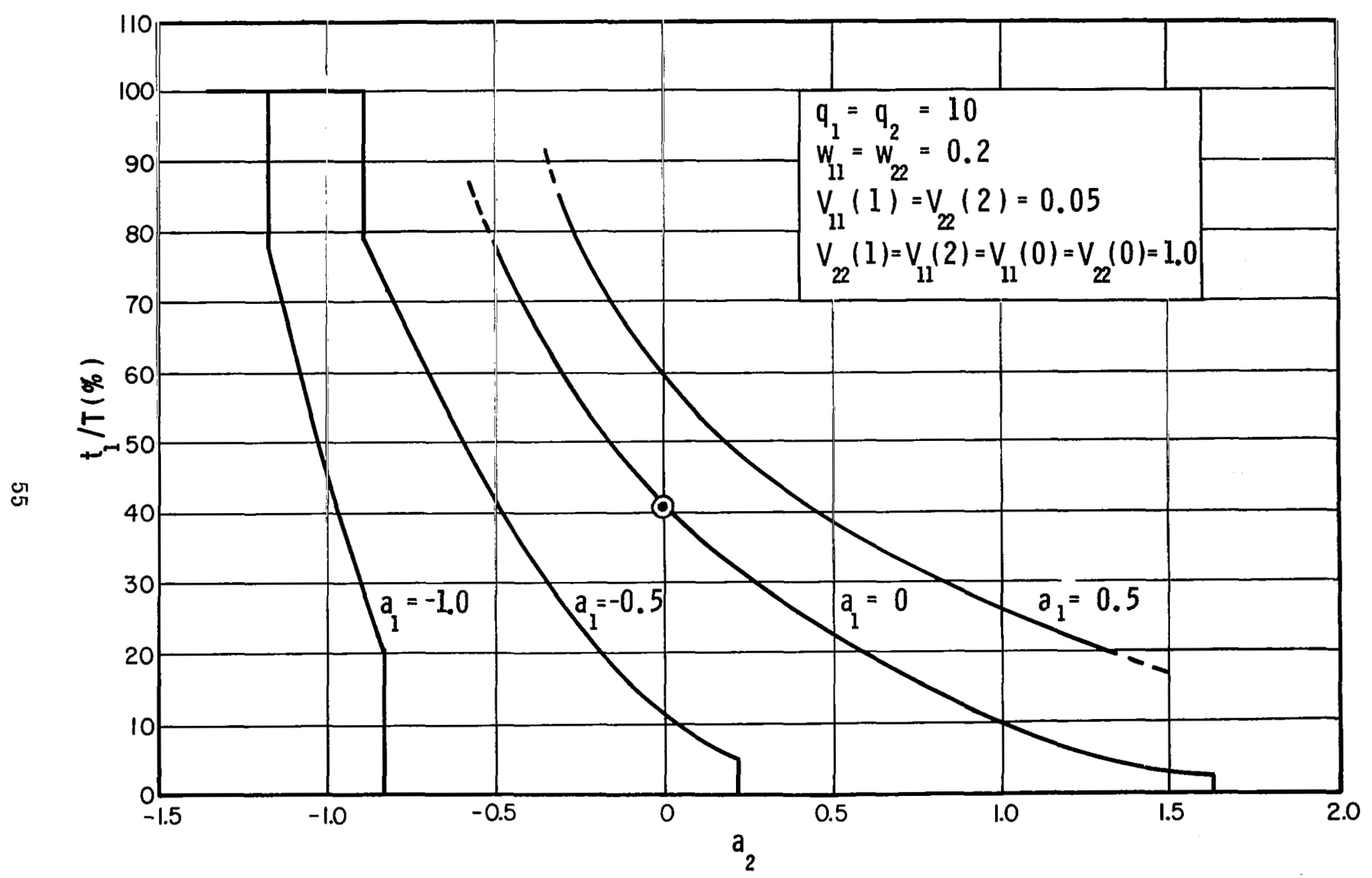

FIG.4a EFFECT OF CONTROLLED ELEMENT DYNAMICS ON FRACTIONAL TIME SPENT ON INSTRUMENT I 


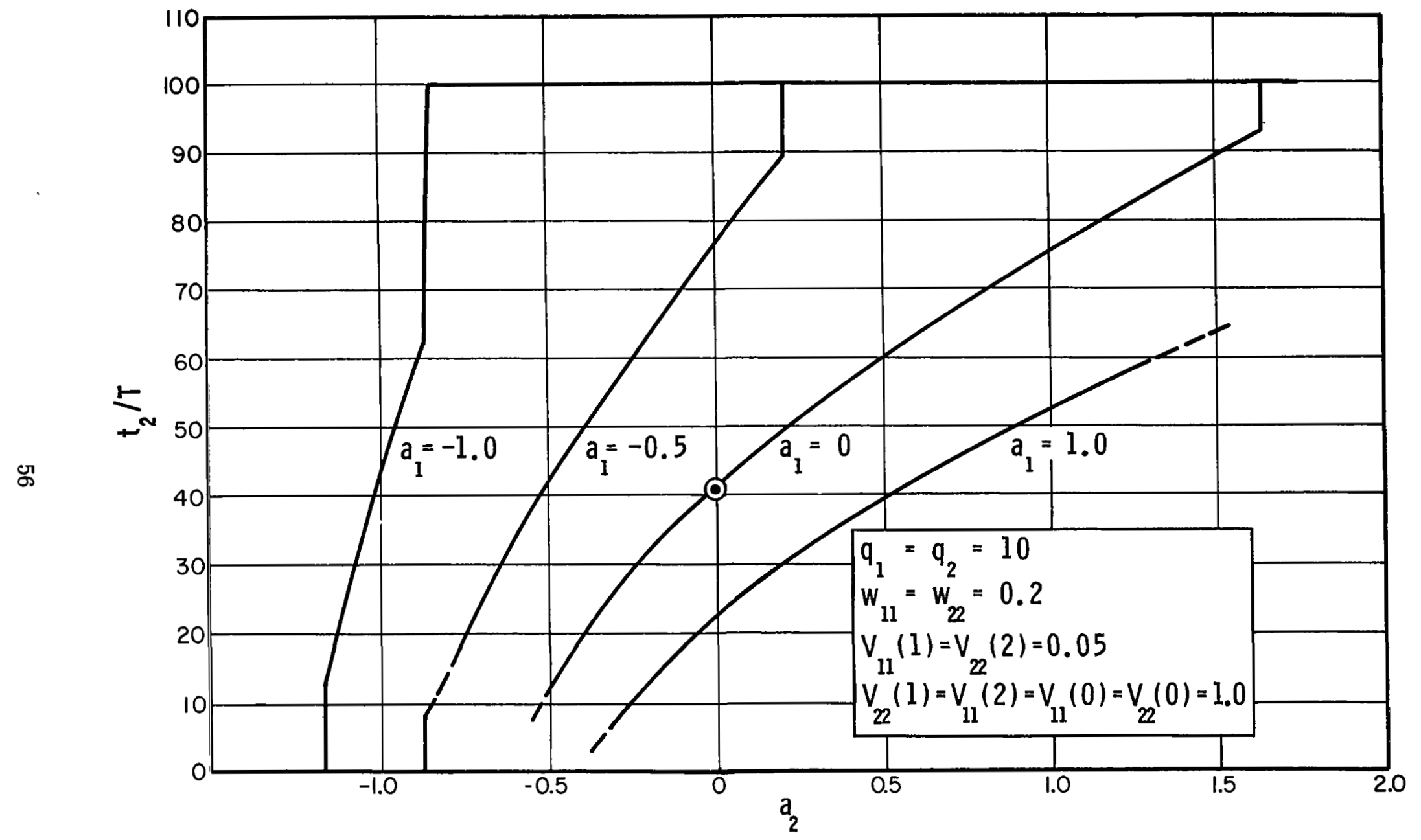

FIG.4b EFFECT OF CONTROLLED ELEMENT DYNAMICS ON FRACTIONAL TIME SPENT ON INSTRUMENT 2 


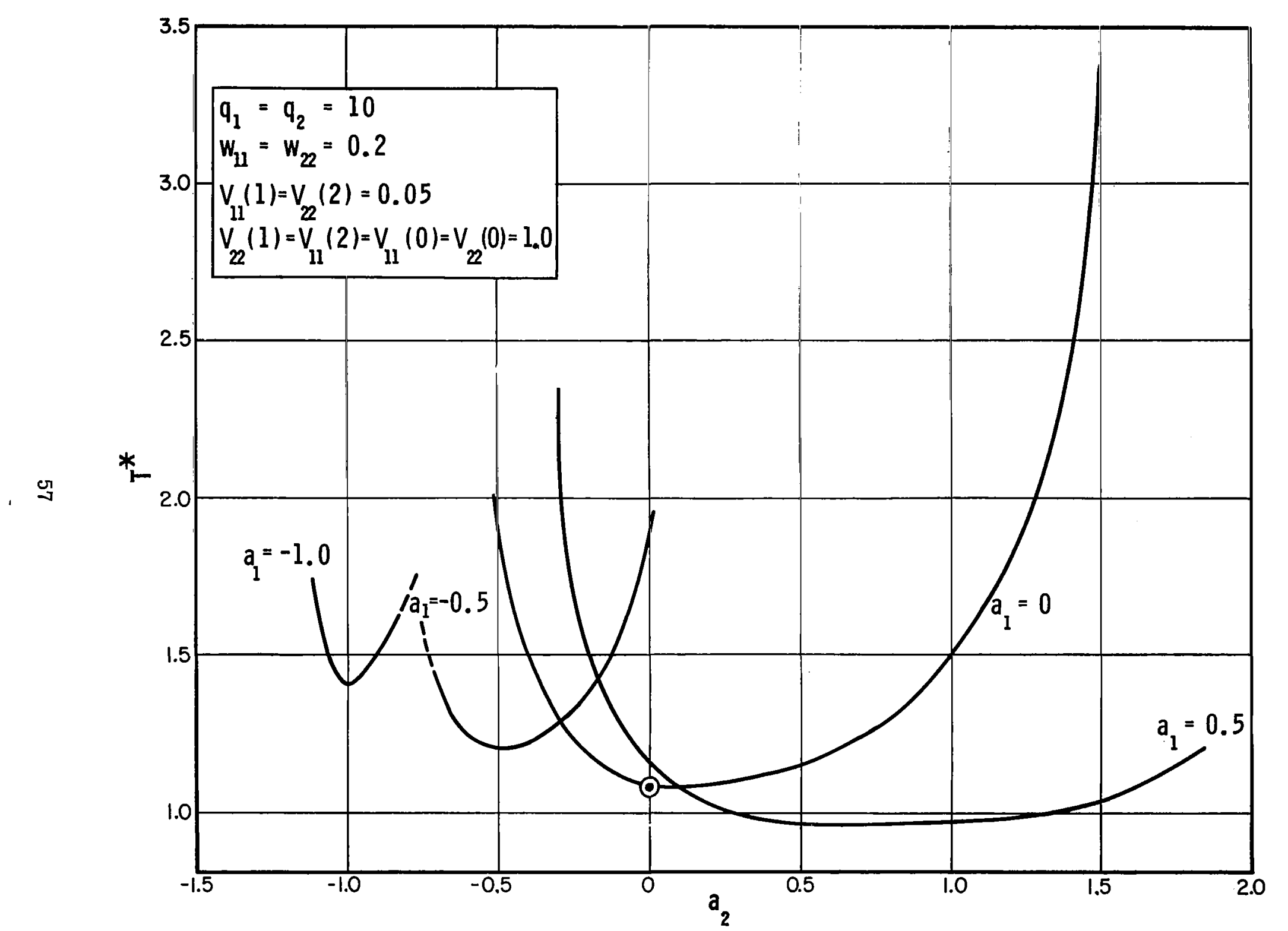

FIG.5 EFFECT OF CONTROLLED ELEMENT DYNAMICS ON OPTIMAL SAMPLING PERIOD 


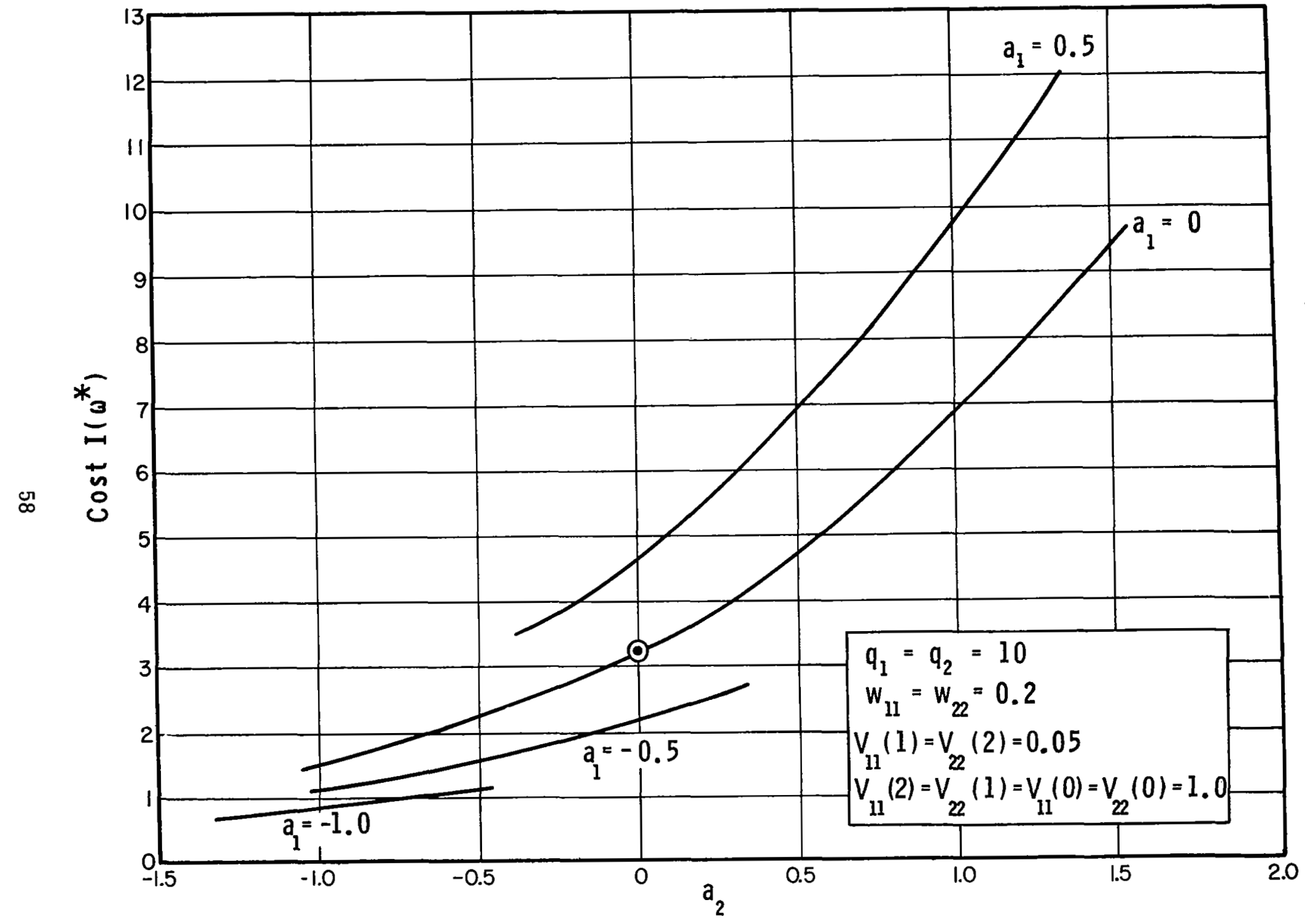

FIG. 6 EFFECT OF CONTROLLED ELEMENT DYNAMICS ON MINIMUM "SAMPLING COST" 


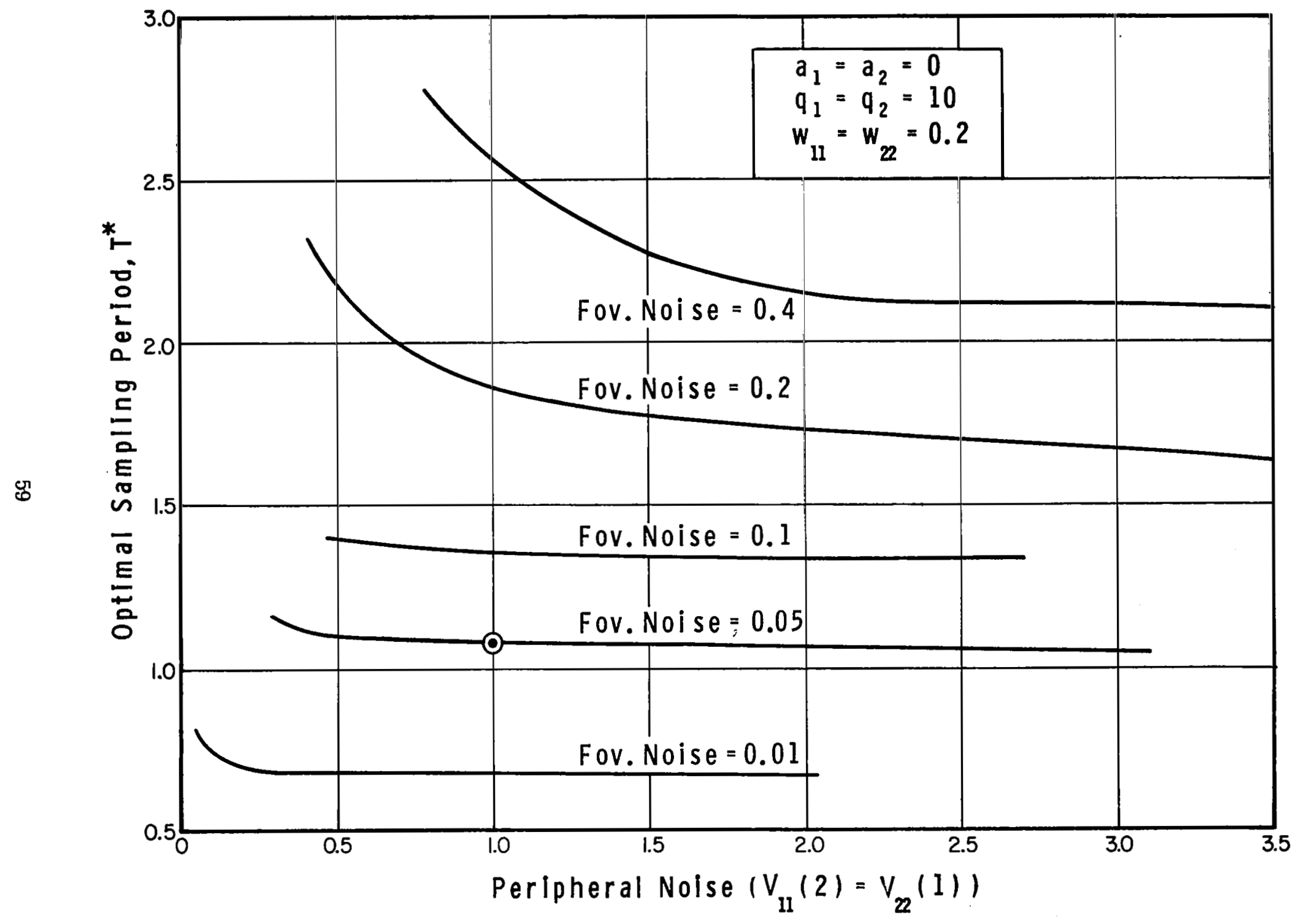

FIG. 7 EFFECT OF OBSERVATION NOISE ON OPTIMAL SAMPLING PERIOD 


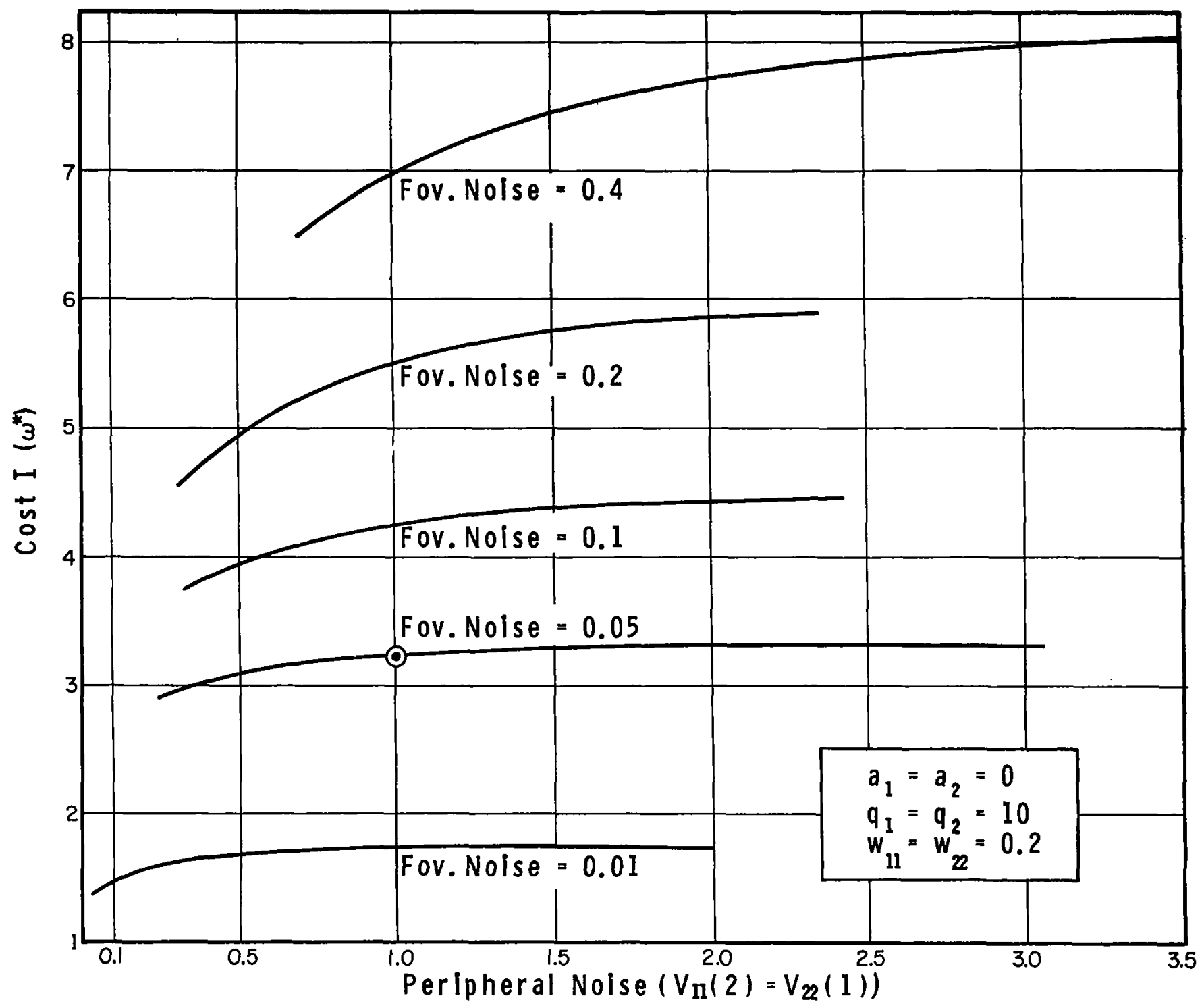

FIG. 8 EFFECT OF OB SERVATION NOISE ON "SAMPLING COSTS" 


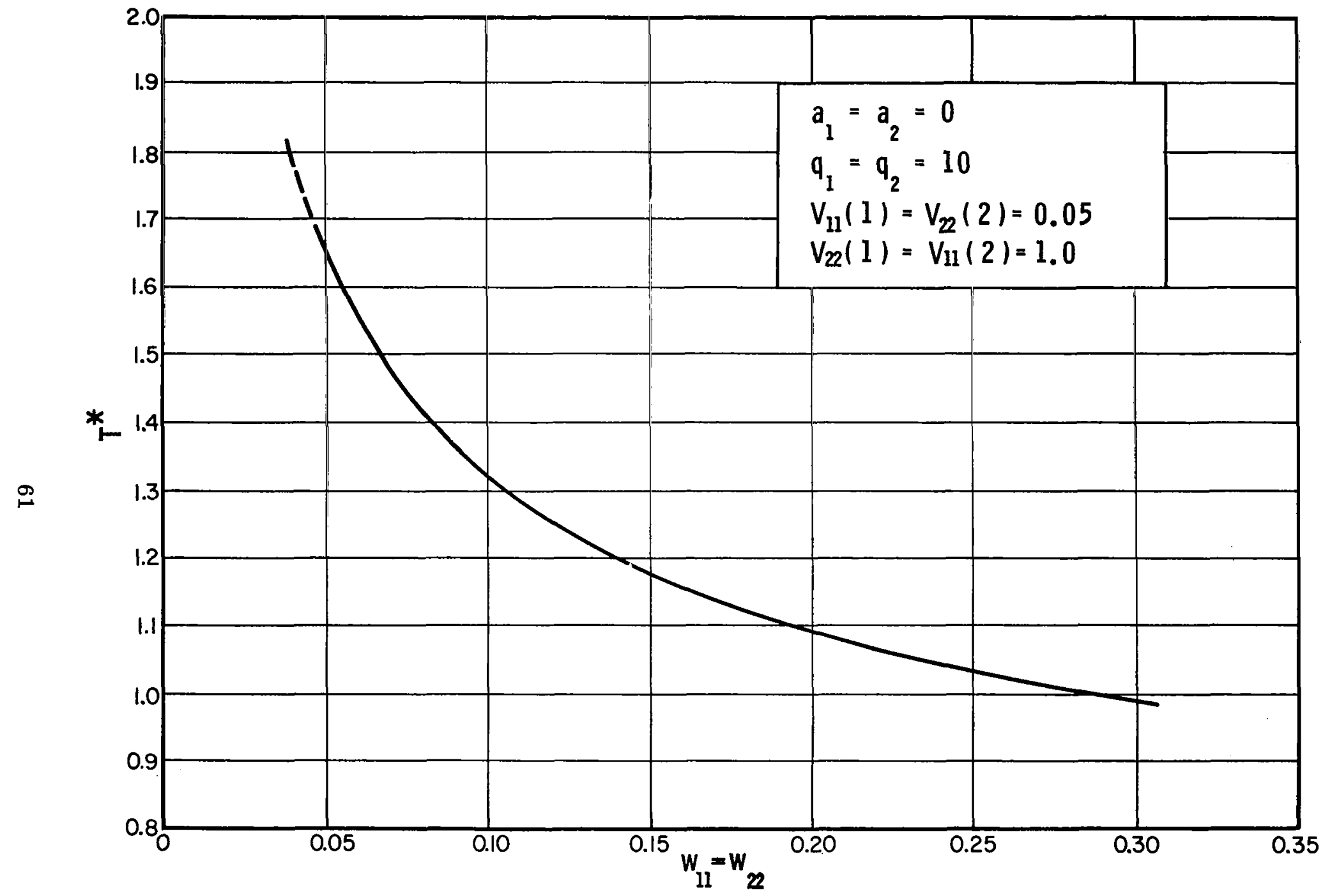

FIG. 9 EFFECT OF INPUT NOISE COVARIANCE ON SAMPLING PERIOD 


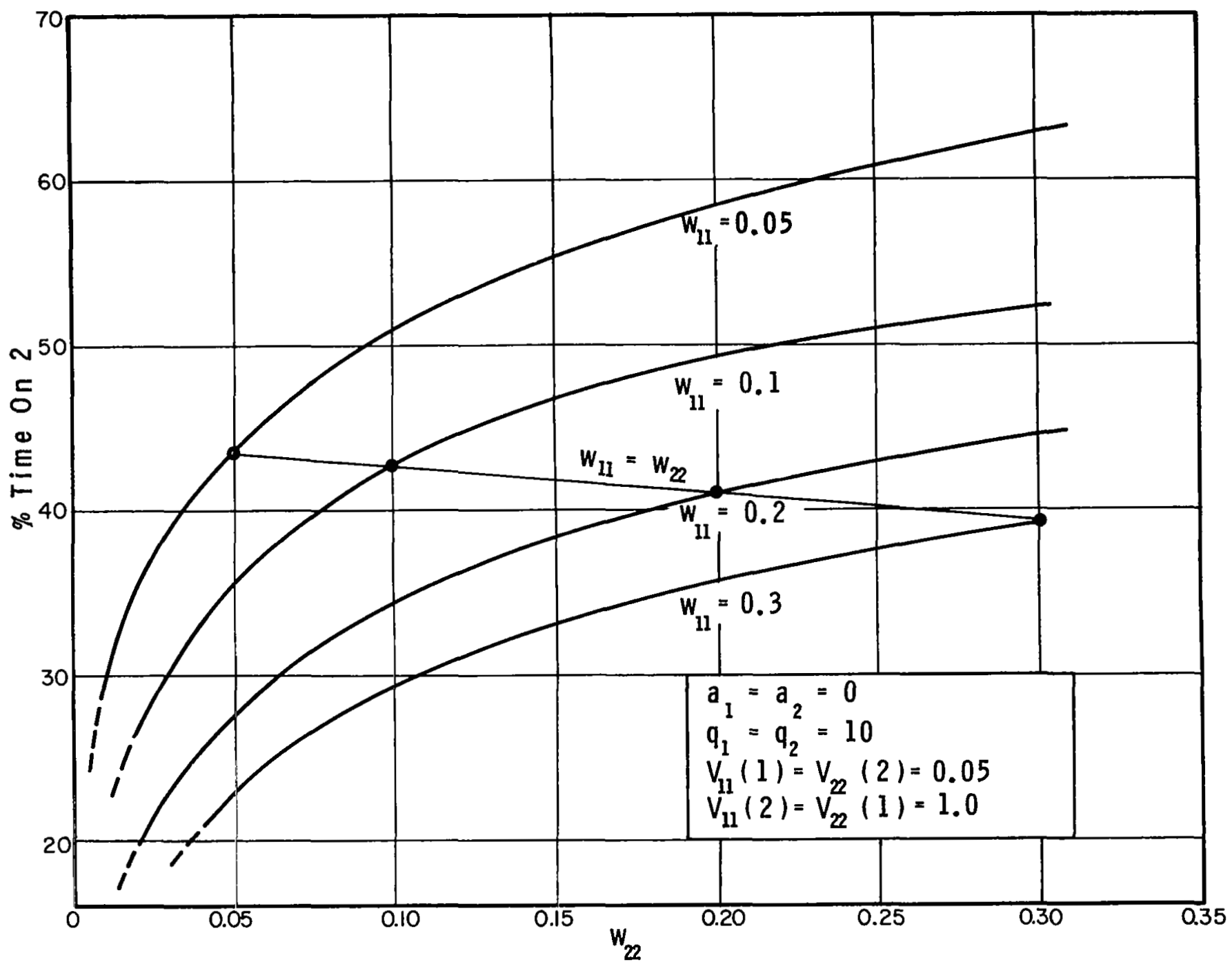

FIG. 10 EFFECT OF INPUT NOISE COVARIANCE ON FRACTIONAL ALLOCATION OF ATTENTION 


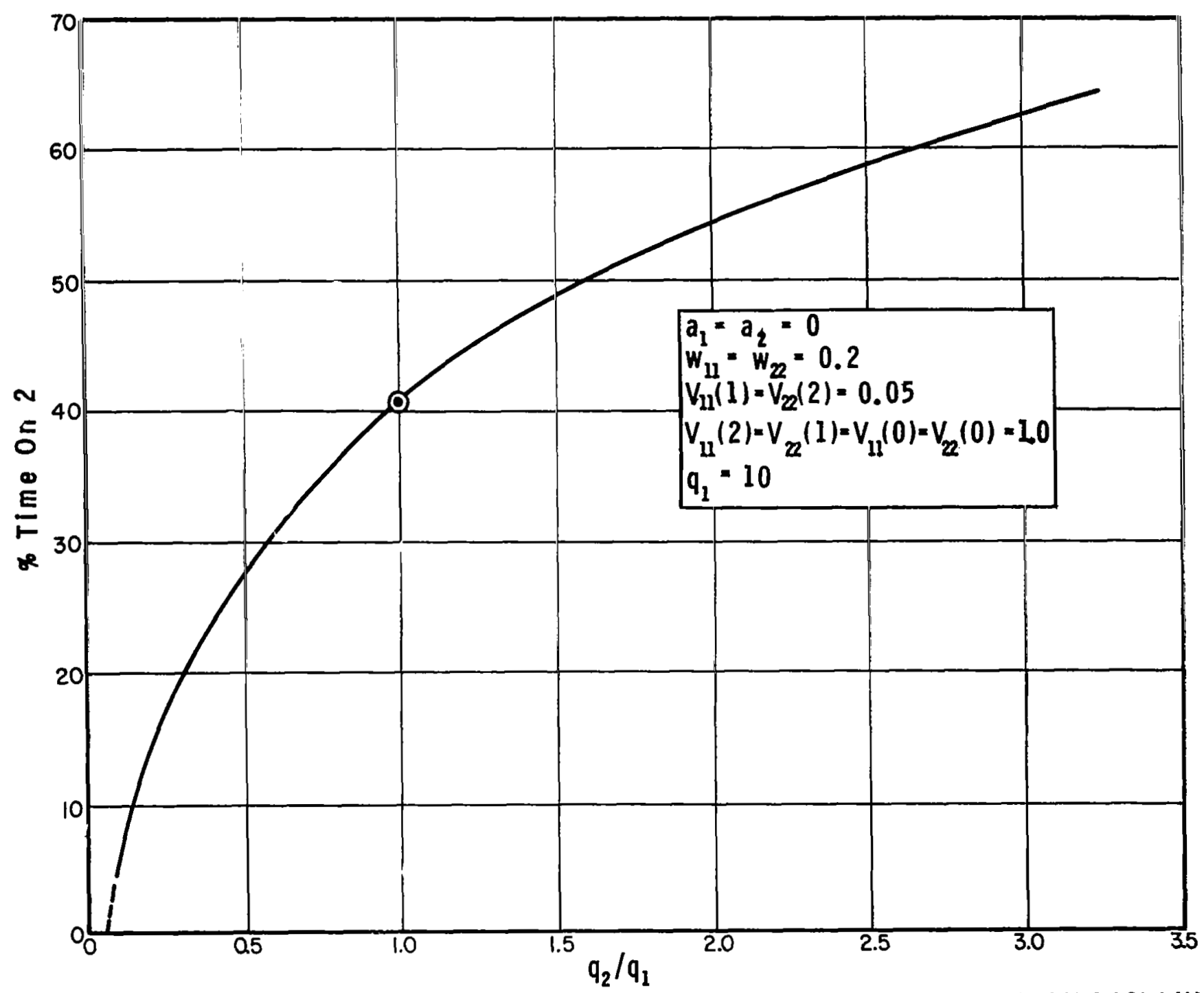

FIG.11 EFFECT OF CONTROL REQUIREMENTS ON FRACTIONAL TIME SPENT ON INSTRUMENT I 


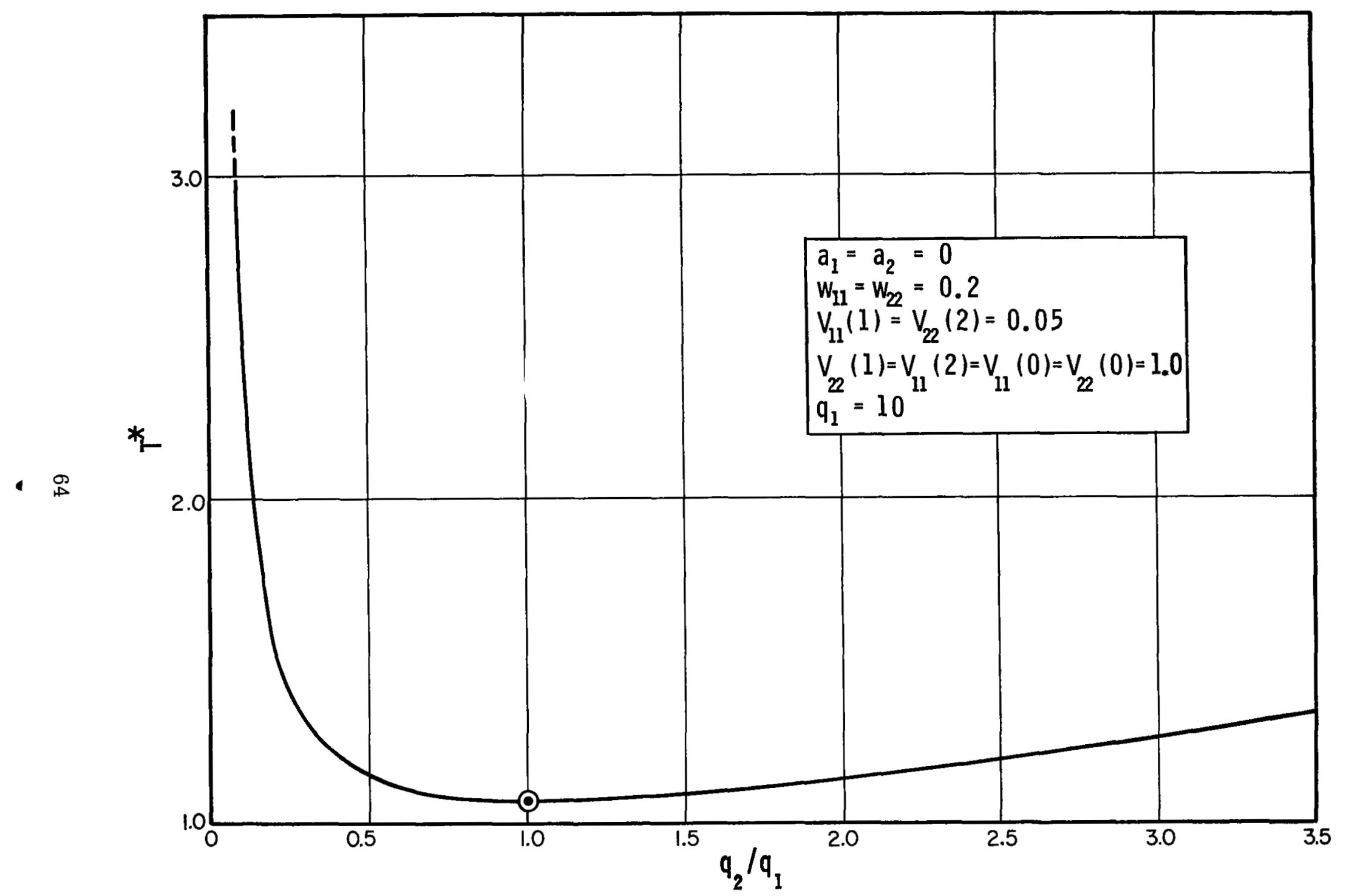

FIG. 12 EFFECT OF CONTROL REQUIREMENTS ON SAMPLING PERIOD 


\section{CONCLUSION}

In order to understand the effects of certain display changes on overall pilot-vehicle system performance, it is necessary to investigate the correlation between control objectives, display properties and various aspects of human behavior. If such an investigation is to result in objective measures of display effectiveness, the interactions among display, pilot and vehicle must be described analytically. This, in turn, requires the development of mathematical models for the various sub-systems, including man himself. In this report we develop a model of the human operator which we feel will be well suited to analytic display evaluation techniques.

The model of the human operator in a multivariable control and monitoring task is developed by considering the human as an information processing-control "system". The key ldea underlying the model is the assumption that the well-trained, well-motivated human operator behaves in a near optimal manner, subject to his inherent limitations. The model contains an element for describIng the human's limitations in addition to representations of his instrument monitoring, data reconstruction and control behavior.

The human's limitations are modelled by combining them into an equivalent perceptual time delay and an equivalent observational nolse. The observation noise covariance depends on where the operator "looks" and, hence, allows the effects of peripheral vision on tracking and Instrument "sampling" behavior to be modelled.

The operator's control behavior is assumed to be similar to that of an ideal feedback controller. The feedback gains, which multiply estimates of the system states, are selected so as to minimize a weighted sum of mean squared tracking errors and control effort. 
It is assumed that the human operator processes the information he obtalns from scanning the display panel so as to reconstruct a "best" estimate of the data necessary for control. The "data reconstructor" consists of a Kalman estimator and a least mean squared predictor in tandem. The structure of the data reconstructor is fixed but its parameters depend on the operator's instrument sampling strategy .

Since an adequate representation of instrument monitoring, or visual sampling, behavior is central to the problem of display evaluation, we devoted most of our attention to this aspect of our human operator model. We reviewed existing sampling models and found that they had certain inherent restrictions which we felt would limit their applicability. Rather than attempt to modify these models, we postulated a new sampling model which was consistent with other portions of our model in that it was based upon an underlying optimization approach. In this approach the operator is viewed as an adaptive-measurement system; he decides where he will direct his foveal visual attention on the basis of a preselected optimality criterion. Th1s criterion depends explicitiy on the control task or control requirements. Peripheral vision is accounted for and there are no restrictions with regard to coupling of signals on various instruments. Provisions for sampling cost and sampling rate constraints are also included in the model.

The assumption of the optimality of the human operator leads to the necessity for solving a multifaceted optimization problem in order to predict specific operator characteristics. We have formulated this problem precisely and have discussed methods for obtaining its solution. Finally, we examined a simple two-axis compensatory tracking problem and used the model to predict instrument sampling behavior. The results exhibit the general 
characteristics one would expect from a human operator performing a similar task.

Some Toplcs for Further Investigation--Thus far, we have postulated a model for the human operator in a controlled environment which has interesting and intuitively appealing properties. We have not, however, verified that this model is a valid representation of human behavior. Previous experiments (Ref. 19) have shown that the controller and predictor portions of the model yield reasonable representations of the human operator. It is now necessary to determine the efflcacy of the sampling and estimation models. Hence, the next step in our research program will be to test these models against existing experimental data.

In the present study the observational noise covariances were chosen arbitrarily. It is our hope that numerical values for these quantities can be measured in appropriate experiments. Since the nolse covariances depend on the display and the environment as well as on Intrinsic human properties, their determination will be extremely difficult. Indeed, it may be necessary to consider the nolse covariances as free parameters which are chosen so as to match experimental data. This would be somewhat unfortunate and it seems that considerable thought should be given to methods for experimentally determining the observation noise.

In addition to the questions concerning the model which must be resolved experimentally, there remain problems of a theoretical nature. In the theoretical development we made several assumptions concerning the separability of various elements in the model. It would be useful to show vigorously under what conditions these assumptions hold. Of greater interest is the examination of methods for mathematically describing the class of sampling strategies $\Omega$. The specification of this class has both mathematical and physical implications. For instance, in the example we 
selected $\Omega$ such that the range space of $\omega(\cdot) \varepsilon \Omega$ was the finite set $\hat{\Omega}=\{0,1,2\}$. Although the results are interpreted in terms of average behavior (because of the infinite interval and the resulting periodicity assumption), this choice of $\Omega$ corresponds, essentially, to defining deterministic sampling strategies. Moreover, this cholce of $\Omega$ introduces mathematical complexities since $\omega$ does not take on a continuum of values. A characterization of $\Omega$ which eliminates this type of complexity and, at the same time, allows for a direct probabilistic interpretation of the optimal sampling strategy $\omega^{*}$ is possible. This, and other, descriptions of the sampling class warrant investigation.

Finally, there is the question of computing algorithms for solving the optimal sampling problem. It seems reasonably clear that the numerical search technique used in the example will prove much too tedious for more complex problems. The investigation of more efficient algorithms thus remains a prime area for research. 


\section{REFERENCES}

1. Burchfiel, J. D., J. I. Elkind and D. C. Miller, "On the Optimal Behavior of the Human Controller: A Pilot Study Comparing the Human Controller with Optimal Control Models," BBN Report No. 1532; August 1967.

2. Jones, R. E., J. L. Milton, and P. M. Fitts, "Eye Fixations of Aircraft Pllots: A Review of Prior Eye-Movement Studies and a Description of a Technique for Recording the Frequency, Duration, and Sequence of Eye Fixations During Instrument Flight," USAF Tech. Report No. 5837 (ATI 65996); September 1949.

3. Milton, J. L., R. E. Jones and P. M. Fitts, "Eye Fixations of Aircraft Pilots: Frequency, Duration, and Sequence of Fixations when Flying the USAF Instrument Low Approach Systems (ILAS)," USAF Tech. Report No. 5839 (ATI 65996); October 1949.

4. Fitts, P. M., R. E. Jones and J. L. Milton, "Eye Fixations of Aircraft Pilots: Frequency, Duration, and Sequence of Fixations when Flying Air Force Ground Controlled Approach System (GCA)," AF Tech. Report No. 5967 (ATI 69038); February 1950 .

5. Jones, R. E., J. L. Milton and P. M. Fitts, "Eye Fixations of Alrcraft Pilots: Frequency, Duration, and Sequence of Fixations During Routine Instrument Flight," AF Tech. Report No. 5975 (ATI 73422); December 1949.

6. Milton, J. L., R. E. Jones and P M. Fitts, "Eye Fixations of Alrcraft Pilots: Frequency, Duration, and Sequence of Fixations when Flying Selected Maneuvers During Instrument and Visual Flight Conditions," AF Tech. Report No. 6018 (ATI 84010); August 1950.

7. Milton, J. L., B. B. McIntosh and F. L. Cole, "Eye Fixations of Aircraft Pilots: Fixations During, Day and Night ILAS Approaches Using an Experimental Instrument Panel Arrangement," USAF Tech. Report No. 6570 (ATI 128350); October 1951.

8. Milton, J. L., B. B. McIntosh and E. L. Cole, "Eye Fixations on Alrcraft Pilots: Fixations During Day and Night GCA Approaches Using an Experimental Instrument Panel Arrangement," USAF Tech. Report No. 6709 (ATI 158203); February 1952. 


\section{REFERENCES (Continued)}

9. Milton, J. L. and F. J. Wolfe, "Eye Fixations of Aircraft Pilots: Fixations During Zero Reader Approaches in a Jet Alrcraft," WADC Tech. Report No. 52-17 (ATI 149050);

February 1952 .

10. Milton, J. L., B. B. MeIntosh and E. L. Cole, "Eye Fixations of Alrcraft Pliots: Routine Maneuvers Under Day and Night Conditions, Using an Experimental Panel Arrangement," WADC Tech. Report No. 53220 (AD 35161); March 1954.

11. Senders, J. W., "The Human Operator as a Monitor and Controller of Multi-Degree of Freedom Systems," IEEE, Transactions on Human Factors in Electronics, HFE-5, No. I, pp. 2-5; September 1964 .

12. Shannon, C. E. and W. Weaver, The Mathematical Theory of Communication, University of Iilinois Press, Urbana, IIIInois.

13. Senders, J. W., J. L. Ward and J.R. Carbonell, "Human V1sual

Sampling Processes: A Simulation Validation Study," BBN Report No. 1485; January 1967.

14. Senders, J. W., J. I. Elkind, M. C. Grignett1 and R. Smallwood, "An Investigation of the Visual Sampling Behavior of Human observers," NASA Report No. CR-434; April 1966.

15. Smallwood, R., "Internal Models and the Human Instrument Monitor," Symposium on Human Factors in Electronics, IEEE, Minneapolis, Minnesota; May 1966.

16. Levison, W. H. and J. I. Elkind, "Studies of Two-Variable Manual Control Systems," JACC Proceedings, University of Pennsylvania, pp. 167-173; June 1967.

17. Carbonell, J. R., "A Queueing Model of Many-Instrument Visual Sampling," IEEE, Transactions on Human Factors in Electronics, Vol. HFE-7, No. 4; December 1966.

18. Senders, J.W., J. L. Ward, J. R. Carbonell, "Human Visual Sampling Processes: Validation Studies in the Operational Flight Trainer," BBN Report No. 1485, Part II; Apr11 1967. 
REFERENCES (Continued)

19. Elkind, J. I., P. L. Falb, D. Kleinman, W. H. Levison, "Optimal Control Theory and Design of Manual Control Systems: A Method for Predicting Control Characteristics and Display Requirements," BBN Report No. 1559; October 1967.

20. McRuer, D. T., D. Graham, E. S. Krendel and W. Reisner, Jr., "Human P1lot Dynamics in Compensatory Systems: Theory, Models and Experiments with Controlled-Element and Forcing Function Variations," AFFDL, TR-65-15; July 1965.

21. Kalman, R. E., "Contributions to the Theory of Optimal Control," Bol. Soc. Mat. Mexicana, pp. 102-119; 1960.

22. Wonham, W. M., "Lecture Notes on Stochast1c Control," Brown University Center for Dynamical Systems, Notes 67-2; 1967.

23. Brockstein, A. J., and B. C. Kuo, "Optimum Control of Multivarlable Sampled Data Systems with Adaptive Sampling," JACC Proceedings, Univ. of Pennsylvania, pp. 366-372; June $1 \overline{967 .}$

24. Jordan, B. W., and E. Polak, "Optimal Control of Aperiodic Discrete Time Systems," J. Siam on Control, Vol. 2, No. 3, pp. 332-346; 1965 .

25. Athans, M., and F. C. Schweppe, "On Optimal Waveform Design via Control Theoretic Concepts," Information and Control, Vol. 10, No. 4, pp. 335-377; 1967 .

26. Meier, L., J. Peschon and R. M. Dressler, "Optimal Control of Measurement Subsystems," JACC Proceedings, Univ. of Pennsylvania, pp. 527-537; June 1967.

27. Kushner, H. J., "On the Optimum Timing of Observations for Linear Control Systems with Unknown Initial State," IEEE Trans. on Auto. Control, Vol. AC-9, No. 2, pp. 144-150; April 1964.

28. Bellman, R. E., "Adaptive Control Processes: A Gulded Tour," Princeton University Press, Princeton, New Jersey; 1961. 


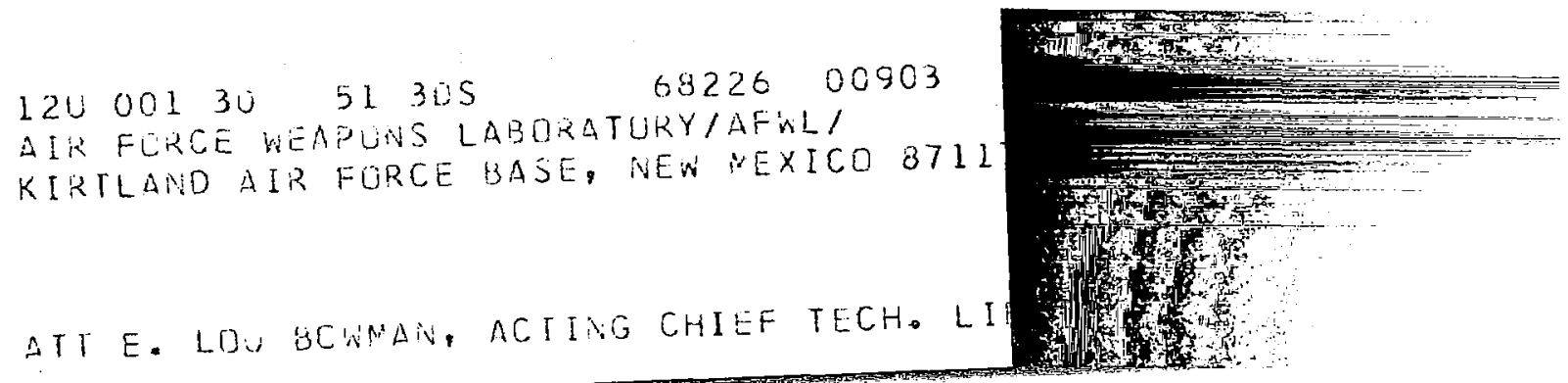

POSTMASTER: If Undeliverable (Seccion 158

"The aeronantical and space activities of the United States shall be conducted so as to contribule... to the expansion of human knowledge of phenomena in the atmosphere and space. The Administration sball provide for the widest practicable and appropriate dissemination of information concerning its activities and the results thereof."

- NAtional AERonautics AND SPACE ACt of 1958

\section{NASA SCIENTIFIC AND TECHNICAL PUBLICATIONS}

TECHNICAL REPORTS: Scientific and technical information considered important, complete, and a lasting contribution to existing knowledge.

TECHNICAL NOTES: Information less broad in scope but nevertheless of importance as a contribution to existing knowledge.

TECHNICAL MEMORANDUMS: Information receiving limited distribution because of preliminary data, security classification, or other reasons.

CONTRACTOR REPORTS: Scientific and technical information generated under a NASA contract or grant and considered an important contribution to existing knowledge.
TECHNICAL TRANSLATIONS: Information published in a foreign language considered to merit NASA distribution in English.

SPECIAL PUBLICATIONS: Information derived from or of value to NASA activities. Publications include conference proceedings, monographs, data compilations, handbooks, sourcebooks, and special bibliographies.

\section{TECHNOLOGY UTILIZATION}

PUBLICATIONS: Information on technology used by NASA that may be of particular interest in commetcial and other non-aerospace applications. Publications include Tech Briefs, Technology Utilization Reports and Notes, and Technology Surveys.

Details on the availability of these publications may be obtained from:

\section{SCIENTIFIC AND TECHNICAL INFORMATION DIVISION}

